\title{
Enhancing sensitivity to neutrino parameters at INO combining muon and hadron information
}

\author{
Moon Moon Devi, ${ }^{a}$ Tarak Thakore, ${ }^{a, b}$ Sanjib Kumar Agarwalla ${ }^{c}$ and Amol Dighe ${ }^{a}$ \\ ${ }^{a}$ Tata Institute of Fundamental Research, \\ Mumbai, 400005 India \\ ${ }^{b}$ Louisiana State University, \\ Baton Rouge, Louisiana, 70803 U.S.A. \\ ${ }^{c}$ Institute of Physics, Sachivalaya Marg, Sainik School Post, \\ Bhubaneswar, 751005 India \\ E-mail: moonmoon4u@tifr.res.in, thakore@phys.1su.edu, \\ sanjib@iopb.res.in, amol@tifr.res.in
}

ABSTRACT: The proposed ICAL experiment at INO aims to identify the neutrino mass hierarchy from observations of atmospheric neutrinos, and help improve the precision on the atmospheric neutrino mixing parameters. While the design of ICAL is primarily optimized to measure muon momentum, it is also capable of measuring the hadron energy in each event. Although the hadron energy is measured with relatively lower resolution, it nevertheless contains crucial information on the event, which may be extracted when taken concomitant with the muon data. We demonstrate that by adding the hadron energy information to the muon energy and muon direction in each event, the sensitivity of ICAL to the neutrino parameters can be improved significantly. Using the realistic detector response for ICAL, we present its enhanced reach for determining the neutrino mass hierarchy, the atmospheric mass squared difference and the mixing angle $\theta_{23}$, including its octant. In particular, we show that the analysis that uses hadron energy information can distinguish the normal and inverted mass hierarchies with $\Delta \chi^{2} \approx 9$ with 10 years exposure at the 50 kt ICAL, which corresponds to about $40 \%$ improvement over the muon-only analysis.

Keywords: Neutrino Physics, Solar and Atmospheric Neutrinos

ARXIV EPRINT: 1406.3689 


\section{Contents}

1 Introduction and motivation 1

2 Methodology 4

2.1 Neutrino interactions and event reconstruction 4

2.2 Binning scheme in $\left(E_{\mu}, \cos \theta_{\mu}, E_{\text {had }}^{\prime}\right)$ space 6

$\begin{array}{lll}2.3 & \text { Details of the numerical analysis } & 7\end{array}$

3 Results with the $\left(E_{\mu}, \cos \theta_{\mu}, E_{\text {had }}^{\prime}\right)$ analysis $\quad 9$

3.1 Identifying the neutrino mass hierarchy 9

$\begin{array}{lll}3.2 & \text { Precision measurement of atmospheric parameters } & 12\end{array}$

$\begin{array}{lll}3.3 \text { Octant of } \theta_{23} & 15\end{array}$

$\begin{array}{lll}4 & \text { Summary and concluding remarks } & 17\end{array}$

\section{Introduction and motivation}

After the recent discovery of a nonzero mixing angle $\theta_{13}$ at reactor $\bar{\nu}_{e}$ disappearance experiments $[1-5]$ and accelerator $\nu_{e} / \bar{\nu}_{e}$ appearance experiments [6-8], the two major remaining unknown issues in neutrino oscillations are (i) whether the neutrino mass hierarchy $(\mathrm{MH})$ is normal $(\mathrm{NH})$ or inverted $(\mathrm{IH})$, i.e. whether $\Delta m_{32}^{2} \equiv m_{3}^{2}-m_{2}^{2}$ is positive or negative, respectively, and (ii) the possible presence of $\mathrm{CP}$ violation $[9,10]$. Here $m_{3}$ corresponds to the neutrino mass eigenstate with the smallest electron component. The moderately large value of $\theta_{13}$ enables us to probe the sub-leading three-flavor effects in current and future neutrino oscillation experiments in order to address these unknowns [11, 12]. In particular, the mass hierarchy, which is a very potent discriminator among models of neutrino mass generation [13] can be probed through the measurement of matter effects [14-18] on neutrinos as they pass through the Earth over long distances. The matter effects induce characteristic differences in the neutrino and antineutrino signals [19, 20], which is the key to unravel the neutrino MH.

The race for the neutrino $\mathrm{MH}$ has received a tremendous boost after the discovery of a moderately large value of $\theta_{13}$. Looking at the current and future neutrino roadmap, a resolution of this issue certainly seems possible in coming ten years or so [21]. Several experimental strategies have been adopted or proposed to determine the type of the neutrino $\mathrm{MH}$. Current generation off-axis long-baseline accelerator experiments T2K [22, 23] and $\mathrm{NO} \nu \mathrm{A}[24-26]$ are expected to provide the first hint of neutrino $\mathrm{MH}[27,28]$ by observing the appearance of $\nu_{e}\left(\bar{\nu}_{e}\right)$ events in a $\nu_{\mu}\left(\bar{\nu}_{\mu}\right)$ beam. Future on-axis superbeam facilities consisting of intense, high power wide-band beams and large next generation detectors, like LBNE [29-33] and LBNO [34-40] due to their relatively longer baselines, can settle this 
issue with much higher confidence. Another interesting test bed for the neutrino MH is the class of medium-baseline reactor experiments, like the proposed JUNO [41] and RENO50 [42]. These future facilities will discriminate between the two different MHs not by using the Earth's matter effect, but through the observation of the interference pattern between the two oscillation frequencies in the reactor antineutrino energy spectrum.

Atmospheric neutrinos can also play a crucial role in this direction. The precise study of atmospheric neutrinos at $\mathrm{GeV}$ energies traveling large distances is enriched with Earth's matter effects which in turn gives information on the neutrino MH [43-48]. The smallness of the atmospheric neutrino flux at $\mathrm{GeV}$ energies can be compensated by using very large detectors, like the low energy extension of IceCube, called PINGU [49] and within the context of the KM3NeT project, a first phase with a dense detector in the open ocean, known as ORCA [50]. Recently, a lot of attention has been given to estimate the MH discovery potential of these proposed facilities [51-58] in light of the large $\theta_{13}$. The proposed magnetized Iron Calorimeter (ICAL) detector located at the India-based Neutrino Observatory (INO) cavern [59,60] is being designed to observe the atmospheric neutrinos at $\mathrm{GeV}$ energies with high detection efficiency and excellent energy and angular resolution for muons $[61,62]$. The most important feature of the ICAL detector is its charge identification capability using a magnetic field which makes it possible to observe $\nu_{\mu}$ and $\bar{\nu}_{\mu}$ events separately. It gives the ICAL detector an edge compared to the other running or proposed atmospheric neutrino experiments and greatly enhances the $\mathrm{MH}$ discovery reach without diluting the Earth's matter effect contained in neutrino and antineutrino signals [63-69].

Though the main focus of ICAL is identification of the neutrino $\mathrm{MH}$, it will also contribute to the precision measurements of the atmospheric neutrino mixing parameters, viz. $\left|\Delta m_{32}^{2}\right|$ and $\theta_{23}$ [70]. One of the major questions here, from the point of view of building models of neutrino mass and mixing [13, 71-73] that try to explain the two large and one relatively small mixing angle in the lepton sector, is whether $\theta_{23}$ is maximal or not, and if it is indeed non-maximal, whether $\theta_{23}$ is less than $45^{\circ}$ (the lower octant - LO solution) or greater than $45^{\circ}$ (the higher octant - HO - solution). This is the so-called problem of octant degeneracy of $\theta_{23}$ [74], which could also be addressed partly by ICAL. This experiment would also be able to put severe constraints on new physics scenarios like $\mathrm{CPT}$ violation [75], and will significantly enhance the reach of $\mathrm{T} 2 \mathrm{~K}$ and $\mathrm{NO} \nu \mathrm{A}$ for detecting $\mathrm{CP}$ violation [76].

ICAL is best suited for observing interactions of $\nu_{\mu}$ and $\bar{\nu}_{\mu}$ from the atmospheric neutrinos, which have energies in the GeV range. When these neutrinos undergo chargedcurrent interactions in the detector, they give rise to muons, which are tracked by the resistive plate chambers (RPCs) that constitute the active component of the detector. The ICAL has been designed to efficiently detect muons of energies in the GeV range, identify their charge, and reconstruct their momenta to a high precision [61,62]. The typical efficiency for detection of a $5 \mathrm{GeV}$ muon travelling vertically is $80 \%$, while the typical charge identification efficiency is more than $95 \%$. The energy $E_{\mu}$ of such a muon can typically be reconstructed with an accuracy of $12 \%$, while its direction may be reconstructed to $1^{\circ}[61,62]$. Owing to this capability, the initial analyses of the physics reach of ICAL have focused on the information from muon energy and direction only [69, 70, 75]. 
However one of the unique features of ICAL is its ability to detect hadron showers and extract information about hadron energy and direction from them. For example, the difference in energies of the interacting neutrino and the outgoing muon, $E_{\text {had }}^{\prime} \equiv E_{\nu}-E_{\mu}$, can be calibrated against the number of hits in the detector due to the hadron shower. The measured number of hits can then be used to reconstruct the fraction of energy of the incoming neutrino that is carried by the hadron. This may be achieved with an energy resolution of $85 \%$ (36\%) for the hadron energy of $1 \mathrm{GeV}(15 \mathrm{GeV})$ [77]. Though the achievable precision on $E_{\text {had }}^{\prime}$ is much lower than that on $E_{\mu}$, it still provides additional information about the particular event, which can be extracted in order to improve the physics reach of the detector. Note that, it is quite challenging to extract the hadronic information at multi-GeV energies in currently running or upcoming water or ice based atmospheric neutrino detectors.

In ICAL, one way of using the hadron information would be to simply add the reconstructed values of $E_{\mu}$ and $E_{\text {had }}^{\prime}$ to reconstruct the energy of the incoming neutrino in each event, which indeed can improve the accuracy in the measurement of $\left|\Delta m_{32}^{2}\right|[78,79]{ }^{1}$ However in the process of adding $E_{\mu}$ and $E_{\text {had }}^{\prime}$ in ICAL, the advantage of high precision in the measurement of $E_{\mu}$ is partially lost in case of $\mathrm{MH}$ discrimination. It has been claimed in [80] that the $\mathrm{MH}$ discovery reach can be improved by treating the reconstructed muon momentum and calibrated $E_{\text {had }}^{\prime}$ as two separate variables. However, since the fraction of neutrino energy carried by the muon, or equivalently the inelasticity $y \equiv E_{\text {had }}^{\prime} / E_{\nu}$, is different for each event, the correlation between these quantities constitutes an important part of the information about the event that should not be missed. This strategy has been suggested earlier in the context of the PINGU and ORCA experiments in [54], where it has been pointed out that by exploring the information on the inelasticity parameter in each event, the $\mathrm{MH}$ reach can be improved by $20-50 \%$. We implement the same idea here in detail in the context of the ICAL experiment to enhance its $\mathrm{MH}$ discrimination capability as well as the precision on the atmospheric parameters.

We therefore adopt the approach of using the values of $E_{\mu}, \cos \theta_{\mu}$, and $E_{\text {had }}^{\prime}$ from each event as independent and correlated pieces of information. In this study, we bin the data in all these three quantities, as opposed to the analyses that use only the muon momentum $\left(E_{\mu}, \cos \theta_{\mu}\right)$. Of course, this also means that the already sparse data has to be further divided into a larger number of bins. Hence we choose to use a slightly coarser binning for $E_{\mu}$ and $\cos \theta_{\mu}$. As will be seen from the results, our approach results in a marked improvement in the ability of the detector to identify the mass hierarchy and increase in the precision on $\left|\Delta m_{32}^{2}\right|$. The magnitude of the improvement is of the same order as was expected in [54].

The structure of the paper is as follows. In section 2 we outline our methodology: extraction of the hadron energy information, the binning scheme, and the $\chi^{2}$ procedure. Section 3 presents the results for the neutino mass hierarchy, precision measurements of the atmospheric oscillation parameters, and for the $\theta_{23}$ octant sensitivity. We conclude in section 4 with a summary of results and comments on our analysis.

\footnotetext{
${ }^{1}$ Such a reconstruction of incoming neutrino energy in multi-GeV range becomes quite difficult in the detectors like Super-Kamiokande due to the poor reconstruction efficiency of multi-ring events; this can be done with a high efficiency only in the sub-GeV range where single-ring events dominate.
} 

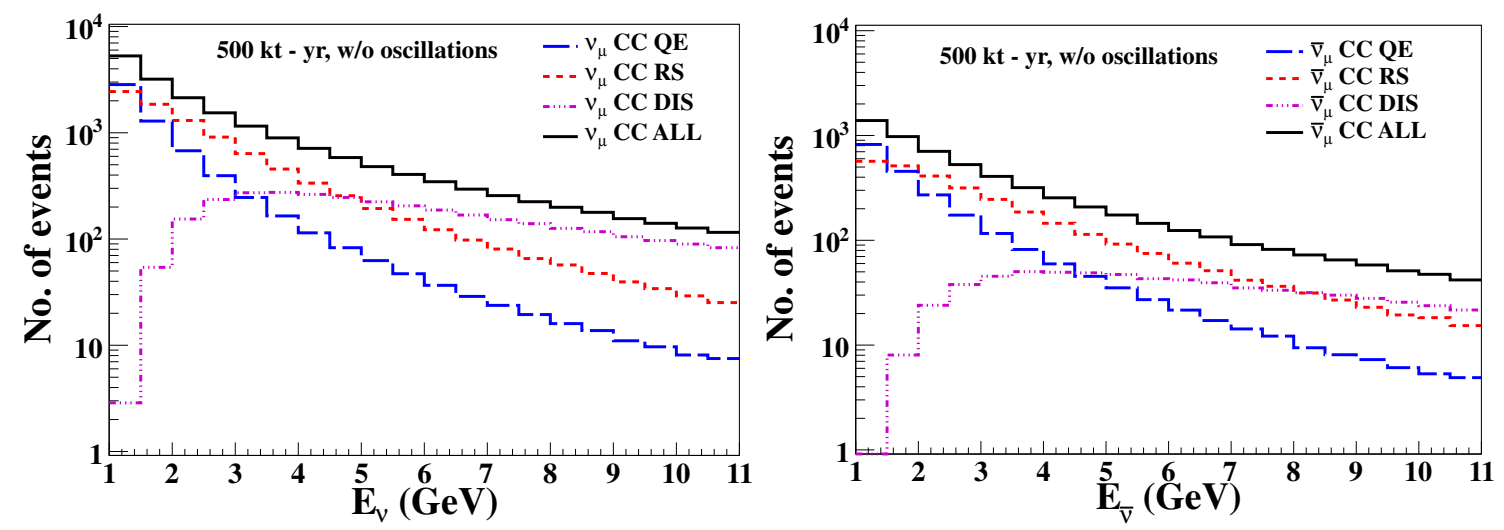

Figure 1. The number of events in the QE, RS and DIS processes at ICAL, as functions of neutrino and antineutrino energies, with an exposure of $500 \mathrm{kt}-\mathrm{yr}$, in the absence of oscillations. The total number of events is also shown.

\section{Methodology}

\subsection{Neutrino interactions and event reconstruction}

The ICAL detector, as described in [59, 62], consists of alternate layers of iron plates and RPCs, which act as the target mass and active detection elements, respectively. When a charged particle passes through an RPC, the $(\mathrm{X}, \mathrm{Y})$ coordinate of its path is recorded in the form of strip hits. The Z-coordinate is provided by the RPC layer number. The hits created by muons in a charged-current $\nu_{\mu}$ interaction give rise to distinct track-like features, while the hits created by hadrons produce shower-like features.

Three main processes contribute to the charged-current $\nu_{\mu}$ interactions in the ICAL detector. In the sub-GeV energy range of neutrinos, the quasi-elastic (QE) process dominates, where the final state muon carries most of the available energy and no hadrons are produced. Hadronic showers make their appearance in resonance (RS) and deep-inelastic scattering (DIS) processes when we move from sub-GeV to multi-GeV range. In the RS process, the final state hadron shower mostly consists of a single pion, though multiple pions may contribute in a small fraction of events. The DIS process produces multiple hadrons, which carry a large fraction of the incoming neutrino energy. figure 1 shows the relative contributions of these three processes to the total number of events in the absence of oscillations, obtained using the event generator NUANCE [81] and the atmospheric neutrino fluxes at Kamioka [82] that we also use in our further analysis in this paper. ${ }^{2}$ It may be observed that in the neutrino energy range of 5 to $10 \mathrm{GeV}$, the contribution of DIS events is significant. This is precisely the energy range where one expects significant matter effects that will help the mass hierarchy identification. The information on hadrons produced in these DIS events is therefore crucial.

The inelasticity in an event, defined as $y \equiv\left(E_{\nu}-E_{\mu}\right) / E_{\nu}=E_{\text {had }}^{\prime} / E_{\nu}$, is roughly the fraction of the neutrino energy that is carried by hadrons. The average inelasticities $\langle y\rangle$

\footnotetext{
${ }^{2}$ Note that the figures 1,2 , and 3 are drawn at the generator level and include the information on the cross section with the target. The detector efficiencies and resolutions are not included.
} 

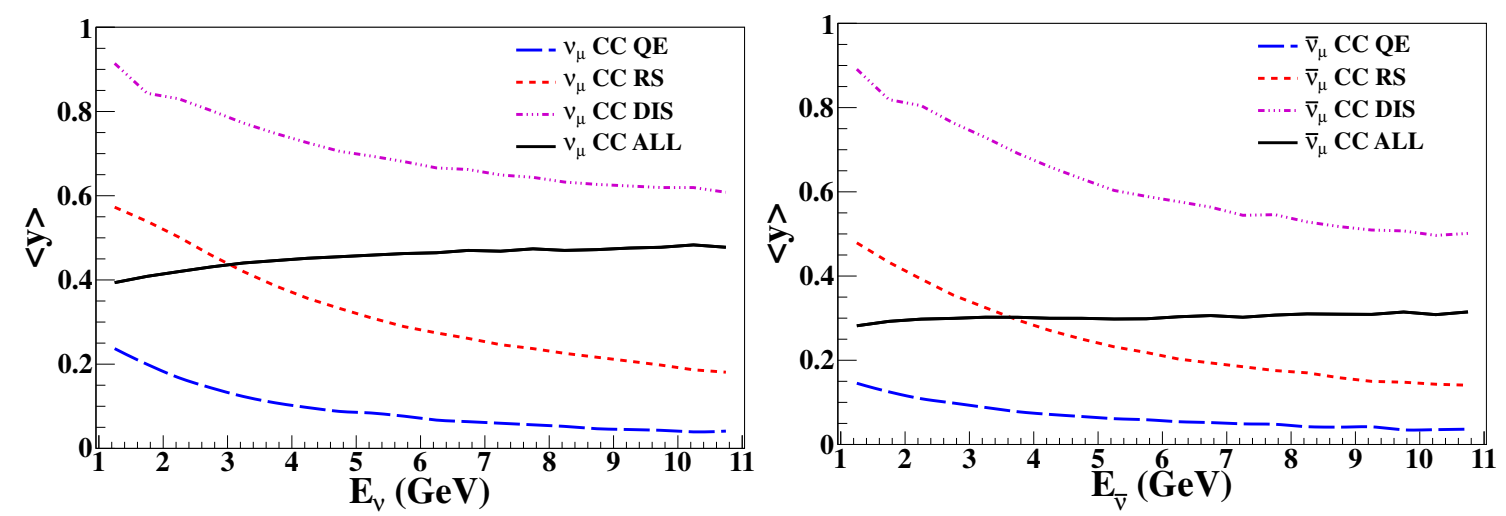

Figure 2. The average inelasticities $\langle y\rangle$ in QE, RS, and DIS processes as a functions of neutrino and antineutrino energies. We also show $\langle y\rangle$ for all channels.
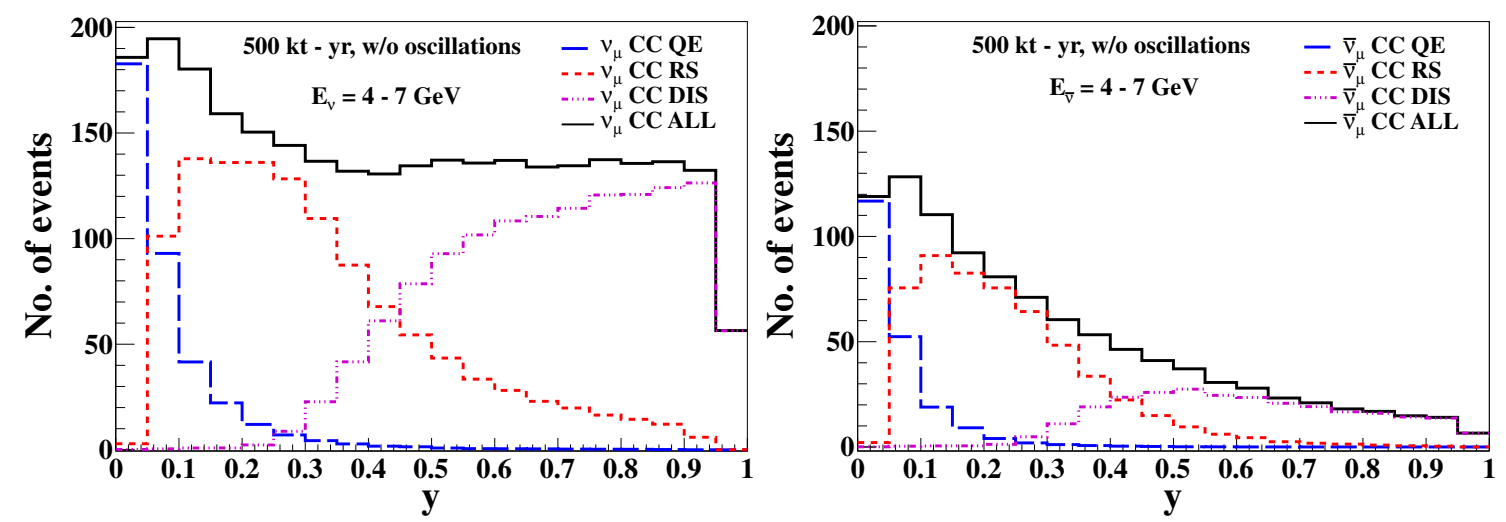

Figure 3. The distribution of inelasticity in events with neutrino and antineutrino energies in the range 4 to $7 \mathrm{GeV}$, with an exposure of $500 \mathrm{kt}-\mathrm{yr}$, in the absence of oscillations.

in the three kinds of processes, as functions of neutrino and antineutrino energies, have been shown in figure 2. Clearly, the average inelasticity in the DIS events is significant, which implies that in the energy range of interest for mass hierarchy identification, a large fraction of the incoming neutrino energy goes into hadrons. While the average inelasticity does not vary much over this energy range, the inelasticities in individual events have a wide distribution (see figure 3). Hence it is important to take into account the $y$ values in individual events. In this paper, we use the energies of hadrons and muons obtained in each event individually, so that the correlation between them is preserved.

We have already mentioned that distinct tracks are created by muon hits and showerlike features emerge from the hadron hits. Figure 4 illustrates a neutrino interaction in the simulated ICAL detector (drawn using the VICE event display package ${ }^{3}$ ) producing a muon track and a hadron shower. The muon reconstruction for ICAL is described in [61], while the hadron reconstruction is described in [77].

In this work, we focus only on the charged-current event where the neutrino interaction produces a muon and possibly also a hadron shower. Note that in general, it may not be

\footnotetext{
${ }^{3}$ See http://www.hecr.tifr.res.in/ samuel/html/vice.html.
} 


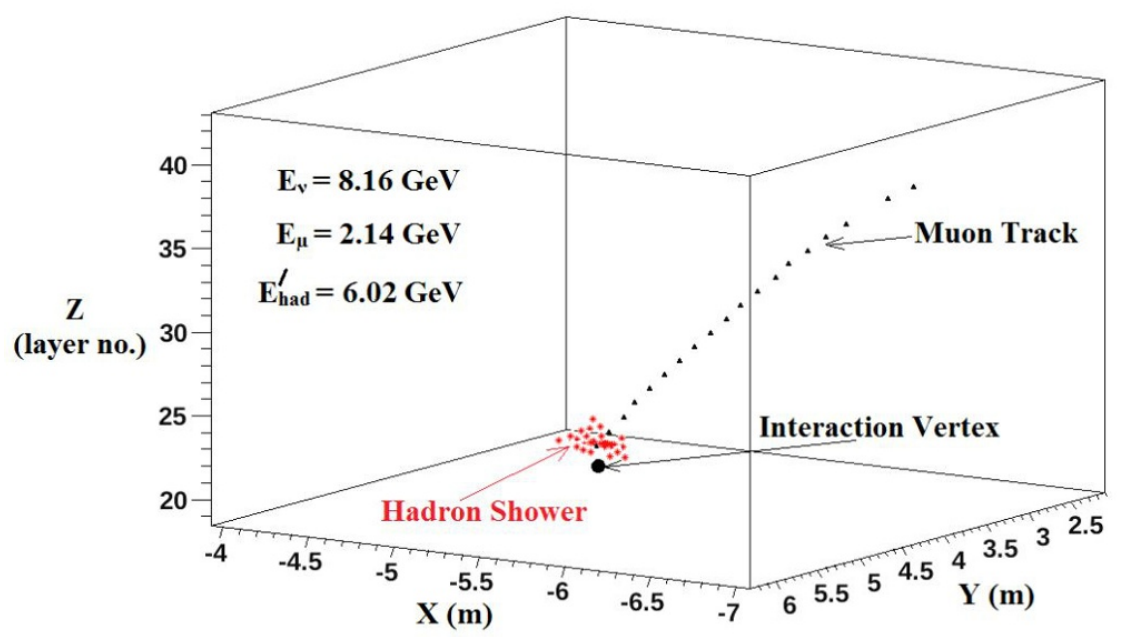

Figure 4. A typical deep-inelastic atmospheric muon-neutrino event in the ICAL detector, obtained using the GEANT4 simulation. Only the relevant part of the detector is shown. X and Y denote length in units of meters whereas $\mathrm{Z}$ represents the layer number.

always possible to distinguish between the muon track and the hadron shower in all events. Here, we assume that the hits created by a muon and hadron can be separated with $100 \%$ efficiency by the ICAL particle reconstruction algorithms. This indeed was also the assumption made while obtaining the muon and hadron response in [61, 77]. Whenever a muon is reconstructed, we take all the other hits to be a part of the hadronic shower for the purpose of hadron energy calibration. This is consistent with the procedure used in [77] for determining the hadron energy calibration. This further implies that the neutrino event reconstruction efficiency is the same as the muon reconstruction efficiency. Note that, the calibration of $E_{\text {had }}^{\prime}$ against the number of hadron shower hits also allows for the possibility of no hits observed in the hadron shower. Finally, the background hits coming from other sources such as the neutral-current events, charged-current $\nu_{e}$ events, cosmic muons, and the noise, have not been taken into account so far. ${ }^{4}$ The systematics due to these effects will have to be taken care of in future, as the understanding of the ICAL detector improves.

\subsection{Binning scheme in $\left(E_{\mu}, \cos \theta_{\mu}, E_{\text {had }}^{\prime}\right)$ space}

After incorporating the reconstruction efficiencies and resolutions for muons and hadrons, in the absence of oscillations one would get about 6200 events with a $\mu^{-}$and 2800 events with a $\mu^{+}$, for an exposure of $500 \mathrm{kt}$-yr. These numbers would decrease further with oscillations. For the analysis presented in [69], 20 uniform $E_{\mu}$ bins in the range 1 to $11 \mathrm{GeV}$ and 80 uniform $\cos \theta_{\mu}$ bins in the range $[-1,+1]$ were used for each polarity of muon. While the excellent energy and angular resolutions of muon in ICAL [61] allow us to use such a fine binning scheme, it does not ensure sufficient statistics for many bins. Including $E_{\text {had }}^{\prime}$ as an additional observable for binning would increase the total number

\footnotetext{
${ }^{4}$ At a magnetized iron neutrino detector (MIND) which is similar to ICAL, the background due to neutral-current events and charged-current $\nu_{e}$ events can be reduced to the level of about a per cent by using the cuts on track quality and kinematics [83].
} 


\begin{tabular}{|c|c|c|c|c|}
\hline Observable & Range & Bin width & Total b & bins \\
\hline$E_{\mu}(\mathrm{GeV})$ & $\begin{array}{c}{[1,4]} \\
{[4,7]} \\
{[7,11]}\end{array}$ & $\begin{array}{c}0.5 \\
1 \\
4\end{array}$ & $\left.\begin{array}{l}6 \\
3 \\
1\end{array}\right\}$ & 10 \\
\hline $\cos \theta_{\mu}$ & $\begin{array}{c}{[-1.0,-0.4]} \\
{[-0.4,0.0]} \\
{[0.0,1.0]}\end{array}$ & $\begin{array}{c}0.05 \\
0.1 \\
0.2\end{array}$ & $\begin{array}{l}12 \\
4 \\
5\end{array}$ & 21 \\
\hline$E_{\text {had }}^{\prime}(\mathrm{GeV})$ & $\begin{array}{c}{[0,2]} \\
{[2,4]} \\
{[4,15]}\end{array}$ & $\begin{array}{c}1 \\
2 \\
11\end{array}$ & $\begin{array}{l}2 \\
1 \\
1\end{array}$ & 4 \\
\hline
\end{tabular}

Table 1. The binning scheme adopted for the reconstructed observables $E_{\mu}, \cos \theta_{\mu}$, and $E_{\text {had }}^{\prime}$ for each muon polarity. The last column shows the total number of bins taken for each observable.

of bins further, reducing the statistical strength of each bin significantly. To avoid this situation we use a coarser binning scheme that is suitable for the three observables $E_{\mu}$, $\cos \theta_{\mu}$, and $E_{\text {had }}^{\prime}$. Now most of the bins have sufficient statistics while at the same time the results are not diluted substantially.

The optimized binning scheme would depend on the parameters one wants to measure. In particular, it could be different for the mass hierarchy identification and precision measurements of atmospheric neutrino mixing parameters. We do not perform an optimization study for binning in this paper, however we identify the regions in the 3-dimensional parameter space $\left(E_{\mu}, \cos \theta_{\mu}, E_{\text {had }}^{\prime}\right)$ that are sensitive to the mass hierarchy, and use finer binning in those regions. These regions roughly span the intervals for $E_{\mu}=4$ to $7 \mathrm{GeV}, \cos \theta_{\mu}=-1$ to -0.4 , and $E_{\text {had }}^{\prime}=0$ to $4 \mathrm{GeV}$. We use coarser binning in other regions. The atmospheric neutrino flux follows a steep power law, resulting in a smaller number of events at higher muon and hadron energies. Therefore, in general, we take finer bins at low energies and wider bins at higher energies, for both muons and hadrons, to ensure sufficient statistics in each bin. This is also consonant with larger uncertainties in energy measurement at higher energies. Our binning scheme is given in table 1. For each polarity, we use 10 bins for $E_{\mu}$, 21 bins for $\cos \theta_{\mu}$, and 4 bins for $E_{\text {had }}^{\prime}$, resulting into a total of $(4 \times 10 \times 21)=840$ bins per polarity.

\subsection{Details of the numerical analysis}

In this analysis, we obtain the physics reach of the ICAL experiment by suppressing the statistical fluctuations of the "observed" event distribution which are simulated by NUANCE in the event rates as well as in the event kinematics. This is implemented by generating events for an exposure of 50,000 kt-yr, followed by incorporating the detector response and then normalizing the event distribution to the actual exposure. This procedure, with the $\chi^{2}$ statistics, is expected to give the median sensitivity of the experiment in the frequentist approach [21]. 
For event generation and inclusion of oscillation, we use the same procedure as described in [69, 70], provisionally using the neutrino fluxes predicted at Kamioka [82]. ${ }^{5}$ The detector response for muons is incorporated by smearing the true muon energy and direction using the Gaussian distributions with the resolution functions obtained from the ICAL detector simulation [61]. The efficiencies of reconstruction and charge identification of muons are also incorporated using the procedure described therein. The hadron energy response is similarly incorporated by smearing the true hadron energy using the Vavilov distribution with the parameters obtained from the fits to the ICAL detector simulations [77]. After incorporating the detector response for muons and hadrons, for the true values of the oscillation parameters as given in table $2\left(\right.$ with $\sin ^{2} 2 \theta_{13}=0.1, \sin ^{2} \theta_{23}$ $=0.5$, and $\mathrm{NH}$ ), one gets about 4500 events with $\mu^{-}$and about 2000 events with $\mu^{+}$for a $500 \mathrm{kt}$-yr exposure. We obtain the distribution of these events in terms of $E_{\mu}, \cos \theta_{\mu}$, and $E_{\text {had }}^{\prime}$.

We define the Poissonian $\chi_{-}^{2}$ for $\mu^{-}$events as:

$$
\chi_{-}^{2}=\min _{\xi_{l}} \sum_{i=1}^{N_{E_{\text {had }}^{\prime}}} \sum_{j=1}^{N_{E_{\mu}}} \sum_{k=1}^{N_{\cos \theta_{\mu}}}\left[2\left(N_{i j k}^{\text {theory }}-N_{i j k}^{\text {data }}\right)-2 N_{i j k}^{\text {data }} \ln \left(\frac{N_{i j k}^{\text {theory }}}{N_{i j k}^{\text {data }}}\right)\right]+\sum_{l=1}^{5} \xi_{l}^{2},
$$

where

$$
N_{i j k}^{\text {theory }}=N_{i j k}^{0}\left(1+\sum_{l=1}^{5} \pi_{i j k}^{l} \xi_{l}\right)
$$

In eq. (2.1), $N_{i j k}^{\text {theory }}$ and $N_{i j k}^{\text {data }}$ are the expected and observed number of $\mu^{-}$events in a given $\left(E_{\mu}, \cos \theta_{\mu}, E_{\text {had }}^{\prime}\right)$ bin. $N_{i j k}^{0}$ are the number of events without systematic errors. Here $N_{E_{\mu}}=10, N_{\cos \theta_{\mu}}=21$, and $N_{E_{\text {had }}^{\prime}}=4$, as mentioned in table 1 . To simulate $N_{i j k}^{\text {data }}$, we have used the oscillation parameters given in table 2 as "true" values. These are benchmark values used in our analysis, and are consistent with those allowed by the global fit [8588]. The effective mass-squared difference is related to the $\Delta m_{31}^{2}$ and $\Delta m_{21}^{2}$ mass-squared differences through the expression [89, 90]:

$$
\Delta m_{\mathrm{eff}}^{2}=\Delta m_{31}^{2}-\Delta m_{21}^{2}\left(\cos ^{2} \theta_{12}-\cos \delta_{\mathrm{CP}} \sin \theta_{13} \sin 2 \theta_{12} \tan \theta_{23}\right) .
$$

The following five systematic errors are included in the analysis using the method of pulls as in [69, 70]: (i) Flux normalization error (20\%), (ii) cross section error (10\%), (iii) tilt error (5\%), (iv) zenith angle error (5\%), and (v) overall systematics (5\%).

Following an identical procedure, $\chi_{+}^{2}$ for $\mu^{+}$events is obtained. Total $\chi^{2}$ is obtained by adding the individual contributions from $\mu^{-}$and $\mu^{+}$events. We also add a $8 \%$ prior (at $1 \sigma$ ) on $\sin ^{2} 2 \theta_{13}$, since this quantity is currently known to this accuracy $[1,2]$. We do not use any prior on $\theta_{23}$ or $\Delta m_{32}^{2}$ since these parameters ${ }^{6}$ will be directly measured at the

\footnotetext{
${ }^{5}$ First calculations of the expected fluxes at the INO site have recently become available [84], and will be implemented in future analysis once they are finalized. The difference in the fluxes at the INO and Kamioka sites arises from the different horizontal components of geomagnetic field at these sites $(40 \mu \mathrm{T}$ at the INO, $30 \mu \mathrm{T}$ at Kamioka).

${ }^{6}$ Adding constraints on these parameters from other experiments will increase the global sensitivity to $\mathrm{MH}$.
} 


\begin{tabular}{|c|c|c|}
\hline Parameter & True value & Marginalization range \\
\hline $\sin ^{2} 2 \theta_{13}$ & $0.09,0.1,0.11$ & {$[0.07,0.11]$} \\
\hline $\sin ^{2} \theta_{23}$ & $0.4,0.5,0.6$ & {$[0.36,0.66]$} \\
\hline$\Delta m_{\mathrm{eff}}^{2}$ & $\pm 2.4 \times 10^{-3} \mathrm{eV}^{2}$ & $\begin{array}{c}{[2.1,2.6] \times 10^{-3} \mathrm{eV}^{2}(\mathrm{NH})} \\
-[2.6,2.1] \times 10^{-3} \mathrm{eV}^{2}(\mathrm{IH})\end{array}$ \\
\hline $\sin ^{2} 2 \theta_{12}$ & 0.84 & Not marginalized \\
\hline$\Delta m_{21}^{2}$ & $7.5 \times 10^{-5} \mathrm{eV}^{2}$ & Not marginalized \\
\hline$\delta_{\mathrm{CP}}$ & $0^{\circ}$ & Not marginalized \\
\hline
\end{tabular}

Table 2. Benchmark oscillation parameters used in this analysis. The second column shows the true values of the oscillation parameters used to simulate the "observed" data set, where the "true value" is the choice of the parameter value for which the data is simulated. The third column shows the range over which the parameter values are varied while minimizing the $\chi^{2}$. This range corresponds to the $3 \sigma$ allowed values of the parameter in the global fit [85-88]. While performing the analysis for precision measurements in section 3.2, we do not marginalize over $\Delta m_{\text {eff }}^{2}$ or $\sin ^{2} \theta_{23}$, and take $\mid \Delta m_{32}^{2}($ true $) \mid=2.4 \times 10^{-3} \mathrm{eV}^{2}$.

ICAL detector. Thus we define:

$$
\begin{aligned}
\chi_{\mathrm{ICAL}}^{2} & =\chi_{-}^{2}+\chi_{+}^{2}+\chi_{\text {prior }}^{2}, \\
\chi_{\text {prior }}^{2} & \equiv\left(\frac{\sin ^{2} 2 \theta_{13}-\sin ^{2} 2 \theta_{13}(\text { true })}{\sigma\left(\sin ^{2} 2 \theta_{13}\right)}\right)^{2} .
\end{aligned}
$$

We take $\sigma\left(\sin ^{2} 2 \theta_{13}\right)=0.08 \times \sin ^{2} 2 \theta_{13}$ (true). While implementing the minimization procedure, $\chi_{\text {ICAL }}^{2}$ is first minimized with respect to the pull variables $\xi_{l}$, and then marginalized over the ranges of oscillation parameters $\sin ^{2} \theta_{23}, \Delta m_{\text {eff }}^{2}$ and $\sin ^{2} 2 \theta_{13}$ as given in table 2 , wherever appropriate. We do not marginalize over $\delta_{\mathrm{CP}}, \Delta m_{21}^{2}$ and $\theta_{12}$ since they have negligible effect on the relevant oscillation probabilities at ICAL [91]. While we use the best-fit values of $\Delta m_{21}^{2}$ and $\theta_{12}$ from the global fit references [85-88], we consider $\delta_{\mathrm{CP}}=0$ throughout our analysis.

\section{Results with the $\left(E_{\mu}, \cos \theta_{\mu}, E_{\text {had }}^{\prime}\right)$ analysis}

In this section, we discuss our findings. We first begin with addressing the neutrino mass hierarchy issue.

\subsection{Identifying the neutrino mass hierarchy}

We quantify the statistical significance of the analysis to rule out the wrong hierarchy by

$$
\Delta \chi_{\mathrm{ICAL}-\mathrm{MH}}^{2}=\chi_{\mathrm{ICAL}}^{2}(\text { false } \mathrm{MH})-\chi_{\mathrm{ICAL}}^{2}(\text { true } \mathrm{MH}) .
$$

Here, $\chi_{\mathrm{ICAL}}^{2}($ true $\mathrm{MH})$ and $\chi_{\mathrm{ICAL}}^{2}($ false $\mathrm{MH}$ ) are obtained by performing a fit to the "observed" data assuming true and false mass hierarchy, respectively. Here with the statistical 


\begin{tabular}{|c|l|l|l|}
\hline$E_{\text {had }}^{\prime}(\mathrm{GeV})$ & events & $\Delta \chi^{2}$ & $\Delta \chi^{2} /$ events \\
\hline $0-1$ & 3995 & 5.8 & 0.0014 \\
\hline $1-2$ & 1152 & 1.9 & 0.0017 \\
\hline $2-4$ & 742 & 1.7 & 0.0023 \\
\hline $4-15$ & 677 & 1.2 & 0.0018 \\
\hline $\begin{array}{c}0-15 \\
\text { (with } E_{\text {had }}^{\prime} \text { information) }\end{array}$ & 6566 & 10.7 & 0.0016 \\
\hline without $E_{\text {had }}^{\prime}$ information & 6775 & 6.3 & 0.0009 \\
\hline
\end{tabular}

Table 3. Contributions of various $E_{\mathrm{had}}^{\prime}$-bins to the total $\Delta \chi^{2}$. The events in the last row without $E_{\text {had }}^{\prime}$ information have true hadron energies up to $100 \mathrm{GeV}$. The same conditions as used for preparing figure 5 have been used here.

fluctuations suppressed, $\chi_{\text {ICAL }}^{2}($ true $\mathrm{MH}) \approx 0$. The statistical significance is also represented in terms of $n \sigma$, where $n \equiv \sqrt{\Delta \chi_{\text {ICAL-MH }}^{2}}$. It has been demonstrated recently [21] that this relation gives the median sensitivity in the frequentist approach of hypothesis testing.

Before presenting the physics reach of ICAL for identifying the $\mathrm{MH}$, we motivate the extent to which the hadron energy information enhances the capability of the experiment for this identification. In figure 5 , we show the distribution of $\Delta \chi_{ \pm}^{2} \equiv \chi_{ \pm}^{2}(\mathrm{IH})-\chi_{ \pm}^{2}(\mathrm{NH})$ in the reconstructed $E_{\mu}-\cos \theta_{\mu}$ plane for the analysis that does not use the hadron energy information (left panels) and the analysis where events are further divided into four subbins depending on the reconstructed hadron energy (right panels). For the sake of this comparison, we do not consider the constant contribution in $\chi^{2}$ coming from the term involving the five pull parameters $\xi_{l}^{2}$ in eq. (2.1). Also, we do not marginalize over the oscillation parameters in the fit. (For our final results, we do take care of the full pull contributions and marginalizations.)

The upper (lower) panels in figure 5 depict the distribution of $\Delta \chi_{-}^{2}\left(\Delta \chi_{+}^{2}\right)$ coming from $\mu^{-}\left(\mu^{+}\right)$events. It can be observed that with the addition of the hadron energy information, the area in the $E_{\mu}-\cos \theta_{\mu}$ plane that contributes significantly to $\Delta \chi_{ \pm}^{2}$ increases, consequently increasing the net $\Delta \chi_{ \pm}^{2}$. Note that this increase in $\chi_{ \pm}^{2}$ is not just due to the information contained in the hadron energy measurement, but also due to that in the correlation between hadron energy and muon momentum.

Another important point to be noted is that the increase in the sensitivity is not simply due to the events with low $E_{\text {had }}^{\prime}$, where the muon energy $E_{\mu}$ could be expected to closely match the original neutrino energy $E_{\nu}$. This may be seen from table 3, where we present the total $\Delta \chi^{2}$ contributions from $\mu^{-}\left(\mu^{+}\right)$events for the four individual hadron bins.

Table 3 shows that, while the $\Delta \chi^{2}$ contribution from the lowest $E_{\text {had }}^{\prime}$ bin is more than half the total $\Delta \chi^{2}$, this bin also has the majority of the total number of events. Indeed, the normalized $\Delta \chi^{2}$ per event (see the last column of table 3 ) is slightly higher for larger $E_{\text {had }}^{\prime}$ bins. This indicates that the hadron energy information from even the higher $E_{\text {had }}^{\prime}$ bins is significant for discriminating between the two mass orderings. 

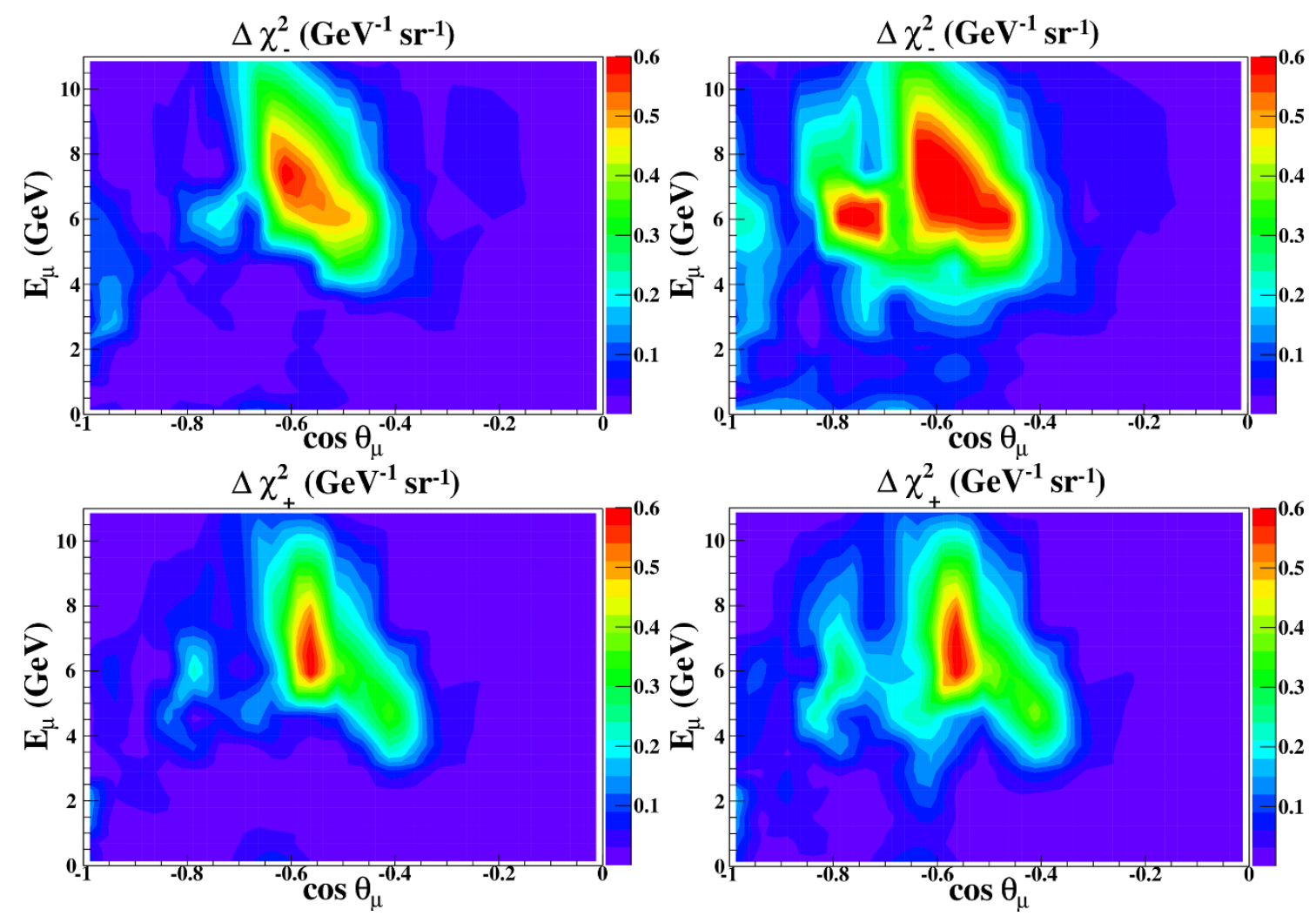

Figure 5. Distribution of $\Delta \chi_{-}^{2}$ per unit area (top panels) and $\Delta \chi_{+}^{2}$ per unit area (bottom panels) in the $\left(E_{\mu}-\cos \theta_{\mu}\right)$ plane, when $\mathrm{NH}$ is taken to be the true hierarchy. The left panels show the distribution when hadron energy information is not used. The right panels show the distribution when hadron energy information is used by further subdividing the events into four hadron energy bins. We take 500 kt-yr of ICAL exposure.

Figure 6 shows the sensitivity of $50 \mathrm{kt}$ ICAL for identifying the neutrino mass hierarchy as a function of the run-time of the experiment. We find that after including the hadron energy information, 10 years of running can rule out the wrong hierarchy with $\Delta \chi_{\text {ICAL-MH }}^{2} \approx$ 9.7 (for true $\mathrm{NH}$ ), and $\Delta \chi_{\mathrm{ICAL}-\mathrm{MH}}^{2} \approx 9.1$ (for true $\mathrm{IH}$ ). Equivalently, the wrong hierarchy can be ruled out to about $3 \sigma$ for either hierarchy. This may be compared with the results without using hadron information. The figure shows that for the same run-time, the value of $\Delta \chi_{\text {ICAL-MH }}^{2}$ increases by about $40 \%$ when the correlated hadron energy information is added. Note that for the comparison here, we have used the same binning scheme in $\left(E_{\mu}, \cos \theta_{\mu}\right)$ as shown in table 1 for both analyses. One may use finer binning for the analysis without hadron information, as has been done in [69]. We find that the $\Delta \chi_{\text {ICAL-MH }}^{2}$ with hadron energy information added is about $35 \%$ more than that in [69]. Thus, the improvement seen in our analysis with hadron energy information is not merely due to using additional bins compared to the muon-only analysis. Here we would like to point out that, in ICAL, we can explore the Earth's matter effect in neutrino and antineutrino channels separately using its charge identification capability via magnetic field. This feature is very crucial in order to enhance the sensitivity to $\mathrm{MH}$. 

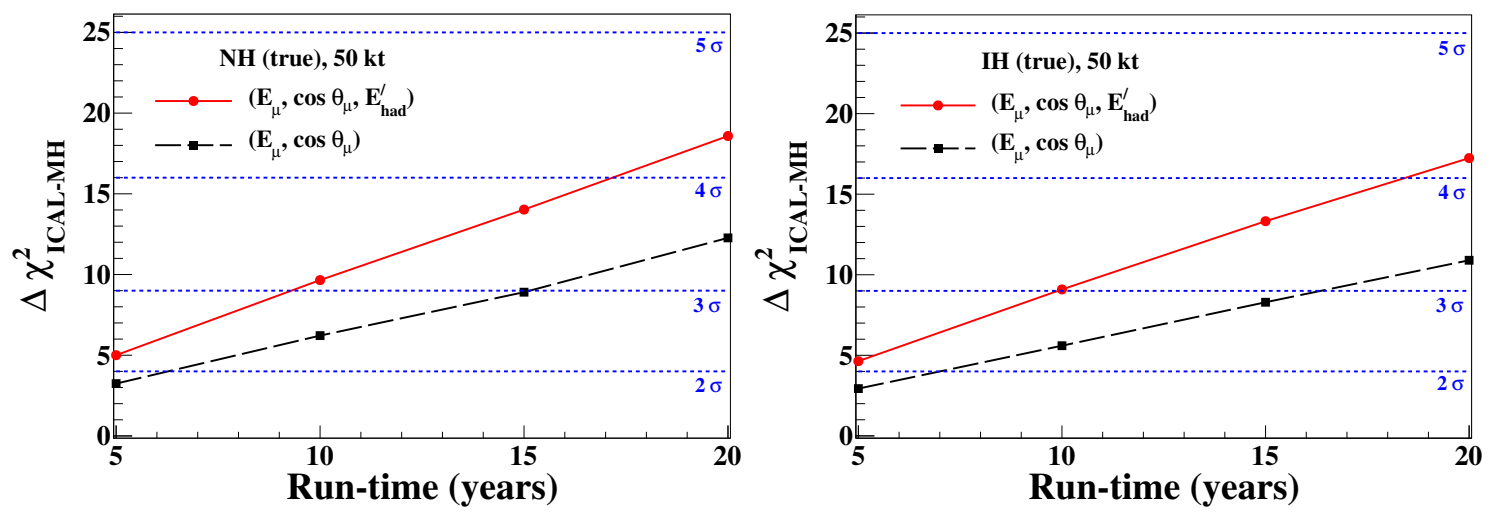

Figure 6. $\Delta \chi_{\mathrm{ICAL}-\mathrm{MH}}^{2}$ as a function of the run-time assuming NH (left panel) and IH (right panel) as true hierarchy. The line labelled $\left(E_{\mu}, \cos \theta_{\mu}\right)$ denotes results without including hadron information, while the line labelled $\left(E_{\mu}, \cos \theta_{\mu}, E_{\mathrm{had}}^{\prime}\right)$ denotes improved results after including hadron energy information. Here we have taken $\sin ^{2} 2 \theta_{13}$ (true) $=0.1$ and $\sin ^{2} \theta_{23}($ true $)=0.5$.

Figures 7 and 8 show the variation of the MH identification potential for three benchmark values of $\sin ^{2} \theta_{23}$ and $\sin ^{2} 2 \theta_{13}$, respectively, in the allowed ranges of these parameters. Higher values of $\sin ^{2} \theta_{23}$ and $\sin ^{2} 2 \theta_{13}$ increase the matter effects in neutrino oscillations and thus result in better hierarchy sensitivity, as seen in these plots. This is expected since the leading matter effect terms in the probability expressions of $P_{\mu \mu}$ and $P_{e \mu}$ are proportional to these parameters [91]. Depending on the true values of these parameters and the true choice of $\mathrm{MH}$, the ICAL detector can identify the $\mathrm{MH}$ with a $\Delta \chi_{\mathrm{ICAL}-\mathrm{MH}}^{2}$ in the range of 7 to 12 using an exposure of 500 kt-yr.

As for the variation of $\Delta \chi_{\text {ICAL-MH }}^{2}$ with respect to $\delta_{\mathrm{CP}}$, we have checked that the projected ICAL atmospheric data is not sensitive to $\delta_{\mathrm{CP}}$, even after the addition of hadron energy information. This is not surprising considering the fact that in the expression of $P_{\mu \mu}$, the $\delta_{\mathrm{CP}}$ dependent term is suppressed by a factor of $\alpha \equiv \Delta m_{21}^{2} / \Delta m_{31}^{2}$ [91]. Recently, this feature of the atmospheric data has also been verified in $[68,69]$.

\subsection{Precision measurement of atmospheric parameters}

In order to quantify the precision in the measurements of a parameter $\lambda$ (here $\lambda$ may be $\sin ^{2} \theta_{23}$ or $\left|\Delta m_{32}^{2}\right|$ or both), we use the quantity:

$$
\Delta \chi_{\operatorname{ICAL}-\mathrm{PM}}^{2}(\lambda)=\chi_{\mathrm{ICAL}}^{2}(\lambda)-\chi_{0}^{2},
$$

where $\chi_{0}^{2}$ is the minimum value of $\chi_{\mathrm{ICAL}}^{2}$ in the allowed parameter range. Here with the statistical fluctuations suppressed, $\chi_{0}^{2} \approx 0$. The significance is denoted by $n \sigma$ where $n \equiv \sqrt{\Delta \chi_{\text {ICAL-PM }}^{2}}$. In terms of these quantities, we define the relative precision achieved on the parameter $\lambda$ at $1 \sigma$ as [92]

$$
p(\lambda)=\frac{\lambda(\max )-\lambda(\min )}{4 \lambda(\text { true })},
$$

where $\lambda(\max )$ and $\lambda(\min )$ are the maximum and minimum allowed values of $\lambda$ at $2 \sigma$ respectively, and $\lambda$ (true) is its true choice. 

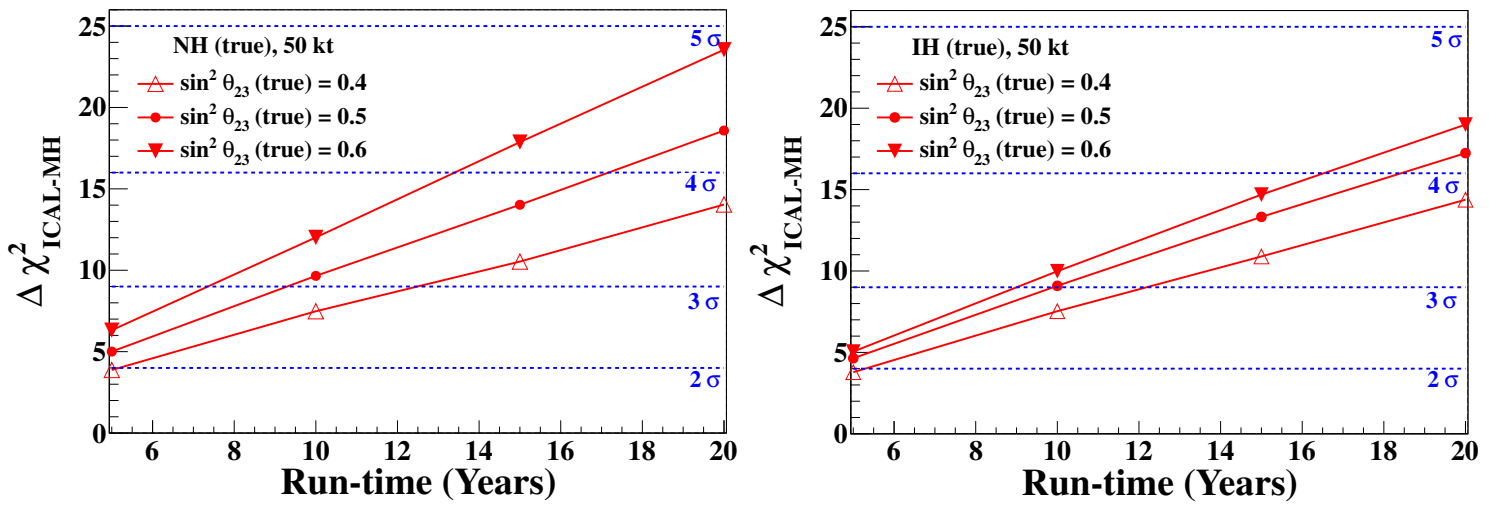

Figure 7. Variation of $\Delta \chi_{\text {ICAL-MH }}^{2}$ for different true values of $\sin ^{2} \theta_{23}$. The left panel (right panel) shows the results assuming $\mathrm{NH}(\mathrm{IH})$ as true hierarchy. Here we have taken $\sin ^{2} 2 \theta_{13}(\operatorname{true})=0.1$.
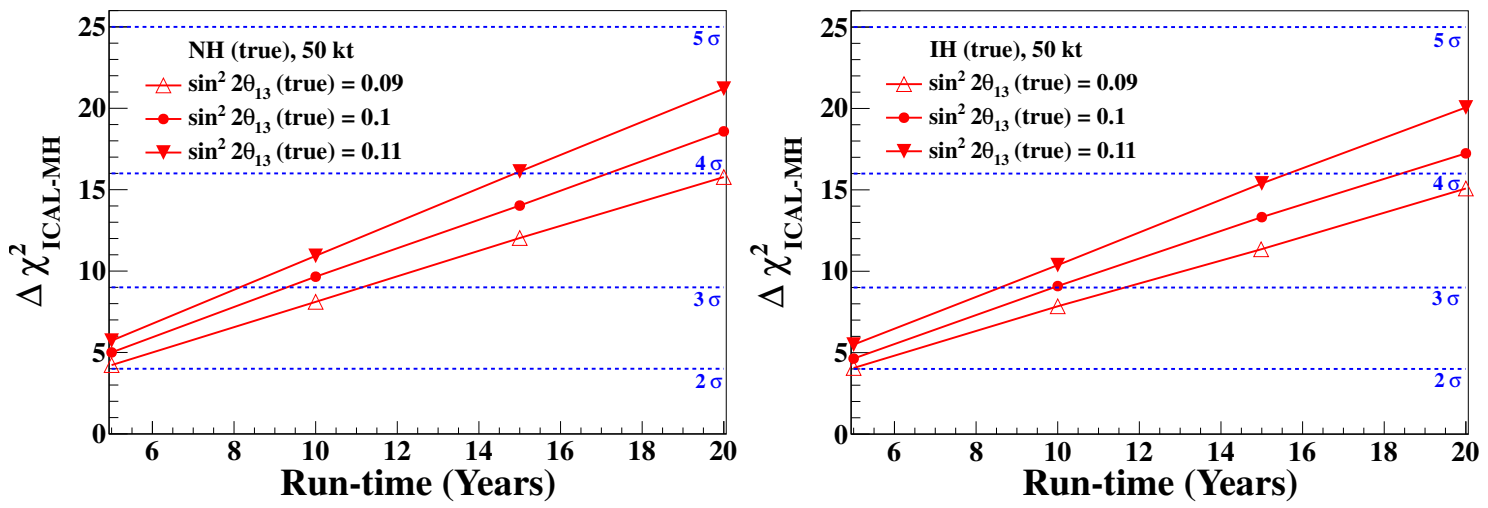

Figure 8. Variation of $\Delta \chi_{\text {ICAL-MH }}^{2}$ for different true values of $\sin ^{2} 2 \theta_{13}$. The left panel (right panel) shows the results assuming $\mathrm{NH}(\mathrm{IH})$ as true hierarchy. Here we have taken $\sin ^{2} \theta_{23}($ true $)=0.5$.

In the two panels of figure 9, we show the sensitivity of ICAL to the two parameters $\sin ^{2} \theta_{23}$ and $\left|\Delta m_{32}^{2}\right|$ separately, where the other parameter has been marginalized over. We also marginalize over $\theta_{13}$ and the two possible choices of mass hierarchies. While the figure shows the results for $\mathrm{NH}$ as the true hierarchy, we have checked that the results with true IH are almost identical. It may be observed from the figure that with the inclusion of hadron energy information, $500 \mathrm{kt}$-yr of ICAL exposure would be able to measure $\sin ^{2} \theta_{23}$ to a relative $1 \sigma$ precision of $12 \%$ and $\left|\Delta m_{32}^{2}\right|$ to $2.9 \%$. With the muon-only analysis, the same relative precisions would be $13.7 \%$ and $5.4 \%$, respectively. Note that, for this comparison, the binning for $\left(E_{\mu}, \cos \theta_{\mu}\right)$ has been kept identical in both scenarios, with and without hadron information. One may use finer binning for the analysis without hadron information. However, we have checked that even the muon-only analysis with finer binning ( $20 E_{\mu}$ bins and $80 \cos \theta_{\mu}$ bins) can yield a precision only up to $13.5 \%$ and $4.2 \%$, respectively, for the $\sin ^{2} \theta_{23}$ and $\left|\Delta m_{32}^{2}\right|$ precision.

The observations above may be understood by noting that the $\sin ^{2} \theta_{23}$ precision is governed mainly by the statistics available to the experiment, which does not change by adding the hadron energy information, and therefore the addition of hadron energy infor- 

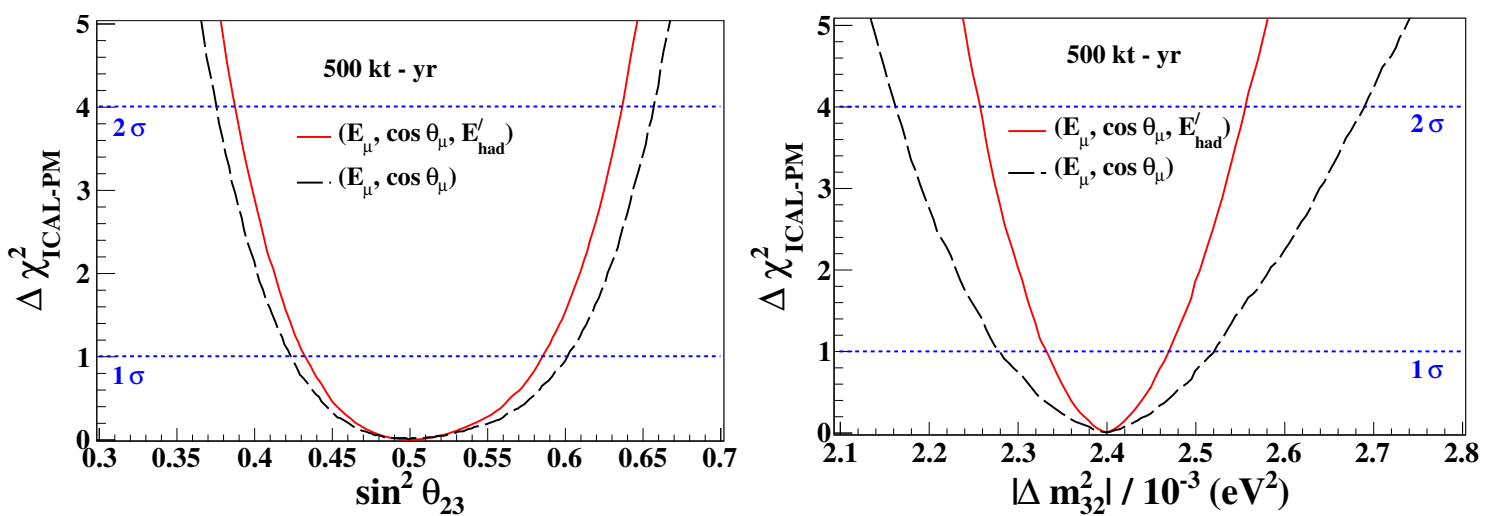

Figure 9. The left panel shows $\Delta \chi_{\text {ICAL-PM }}^{2}\left(\sin ^{2} \theta_{23}\right)$ and the right panel depicts $\Delta \chi_{\text {ICAL-PM }}^{2}\left(\left|\Delta m_{32}^{2}\right|\right)$, assuming $\mathrm{NH}$ as true hierarchy. The lines labelled $\left(E_{\mu}, \cos \theta_{\mu}\right)$ denote results without including hadron information, while the lines labelled $\left(E_{\mu}, \cos \theta_{\mu}, E_{\mathrm{had}}^{\prime}\right)$ denote improved results after including hadron energy information.
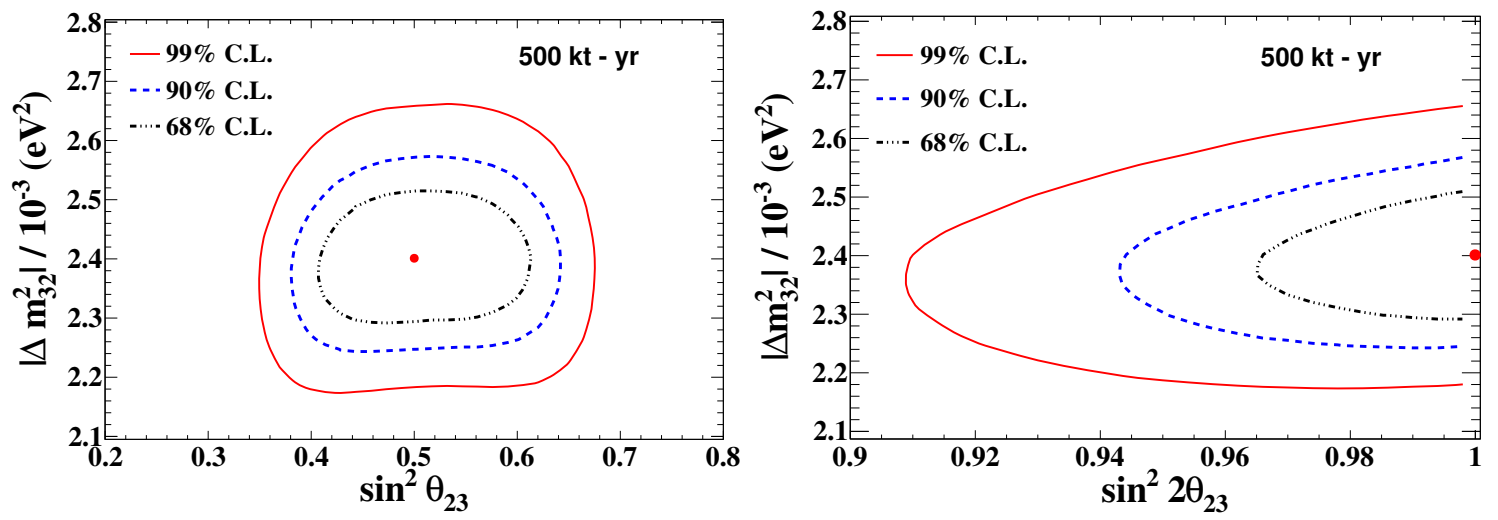

Figure 10. $\Delta \chi_{\text {ICAL-PM }}^{2}$ contours at $68 \%, 90 \%$, and $99 \%$ confidence levels ( 2 dof) in $\sin ^{2} \theta_{23^{-}}$ $\left|\Delta m_{32}^{2}\right|$ plane (left panel) and in $\sin ^{2} 2 \theta_{23}-\left|\Delta m_{32}^{2}\right|$ plane (right panel) after including the hadron energy information. We assume $\mathrm{NH}$ as the true hierarchy. The true choices of the parameters have been marked with a dot.

mation makes only a small difference in the two analyses. On the other hand, independent measurements of $E_{\mu}$ and $E_{\text {had }}^{\prime}$ corresponds to a better estimation of $E_{\nu}$, which appears in the oscillation expression as $\sin ^{2}\left(\Delta m^{2} L / E_{\nu}\right)$. A better measurement of $E_{\nu}$ thus leads to a better measurement of $\Delta m^{2}$, resulting in the dramatic improvement in the precision on $\Delta m_{32}^{2}$ observed here.

Figure 10 shows the $\Delta \chi_{\text {ICAL-PM }}^{2}$ contours at $68 \%, 90 \%$, and $99 \%$ confidence levels in the $\sin ^{2} \theta_{23}-\left|\Delta m_{32}^{2}\right|$ plane (left panel) and in the $\sin ^{2} 2 \theta_{23}-\left|\Delta m_{32}^{2}\right|$ plane (right panel) after including the hadron energy information. Here the true value of $\theta_{23}$ has been taken to be maximal, so the contours in the left panel are almost symmetric in $\sin ^{2} \theta_{23}$. The comparison of the projected $90 \%$ C.L. precision reach of ICAL (500 kt-yr exposure) in $\sin ^{2} \theta_{23}-\left|\Delta m_{32}^{2}\right|$ plane with other experiments [93-95] is shown in figure 11. Using hadron energy information, the ICAL will be able to achieve a $\sin ^{2} \theta_{23}$ precision comparable to the current precision for Super-Kamiokande [93] or T2K [95], and the $\left|\Delta m_{32}^{2}\right|$ precision comparable to 


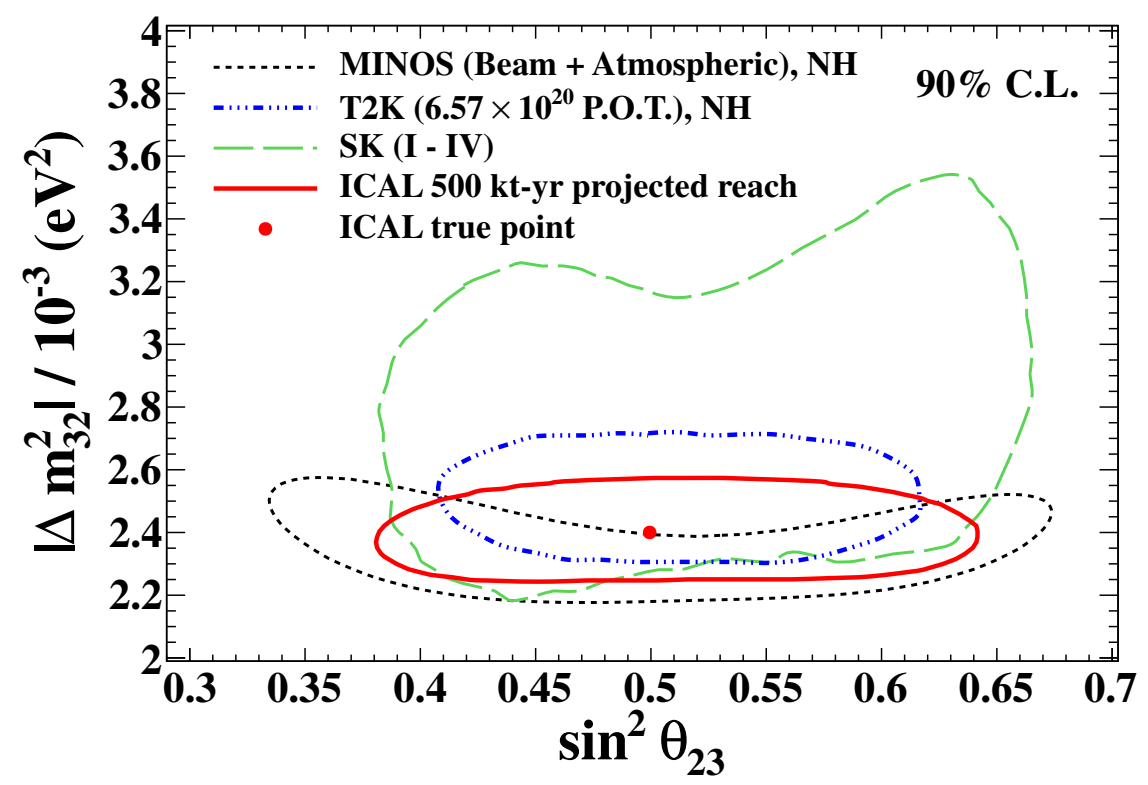

Figure 11. 90\% C.L. (2 dof) contours in the $\sin ^{2} \theta_{23}-\left|\Delta m_{32}^{2}\right|$ plane. The current limits from SuperKamiokande [93], MINOS [94], and T2K [95] have been shown along with the projected ICAL reach for the exposure of $500 \mathrm{kt}-\mathrm{yr}$, assuming true NH. The true choices of the parameters for ICAL have been marked with a dot.

the MINOS reach [94]. Of course, some of these experiments would have collected much more statistics by the time ICAL would have an exposure of $500 \mathrm{kt}-\mathrm{yr}$. The ICAL will therefore not be competing with these experiments for the precision measurements of these mixing parameters, however the ICAL measurements will serve as complementary information for the global fit of world neutrino data. Note that, as compared to the atmospheric neutrino analysis at Super-Kamiokande, the ICAL precision on $\left|\Delta m_{32}^{2}\right|$ is far superior. This is a consequence of the better precision in the reconstruction of muon energy and direction at ICAL.

Finally in this subsection, we present $68 \%, 90 \%$, and $99 \%$ C.L. contours in the $\sin ^{2} \theta_{23}-$ $\left|\Delta m_{32}^{2}\right|$ plane, considering non-maximal values of the mixing angle $\theta_{23}$. Figure 12 shows the sensitivity of ICAL for $\sin ^{2} 2 \theta_{23}=0.93$ (i.e. $\sin ^{2} \theta_{23}=0.37,0.63$ ). It can be seen that the precisions obtained are similar, though the shapes of the contours are more complicated. We observe that for $\theta_{23}$ in the lower octant, the maximal mixing can be ruled out with $99 \%$ C.L. with $500 \mathrm{kt}-\mathrm{yr}$ of ICAL data. However, if $\theta_{23}$ is closer to the maximal mixing value, or in the higher octant, then the ICAL sensitivity to exclude maximal mixing would be much smaller. These contours can also be seen as precursors to resolving the $\theta_{23}$ octant degeneracy, which will be discussed in section 3.3.

\subsection{Octant of $\theta_{23}$}

One can exploit the Earth's matter effect in the $P_{\mu \mu}$ channel to resolve the octant ambiguity of $\theta_{23}$ [96]. In analogy with the mass hierarchy discovery potential, we quantify the statistical significance of the analysis to rule out the wrong octant by

$$
\Delta \chi_{\text {ICAL-OS }}^{2}=\chi_{\text {ICAL }}^{2}(\text { false octant })-\chi_{\text {ICAL }}^{2}(\text { true octant }) .
$$



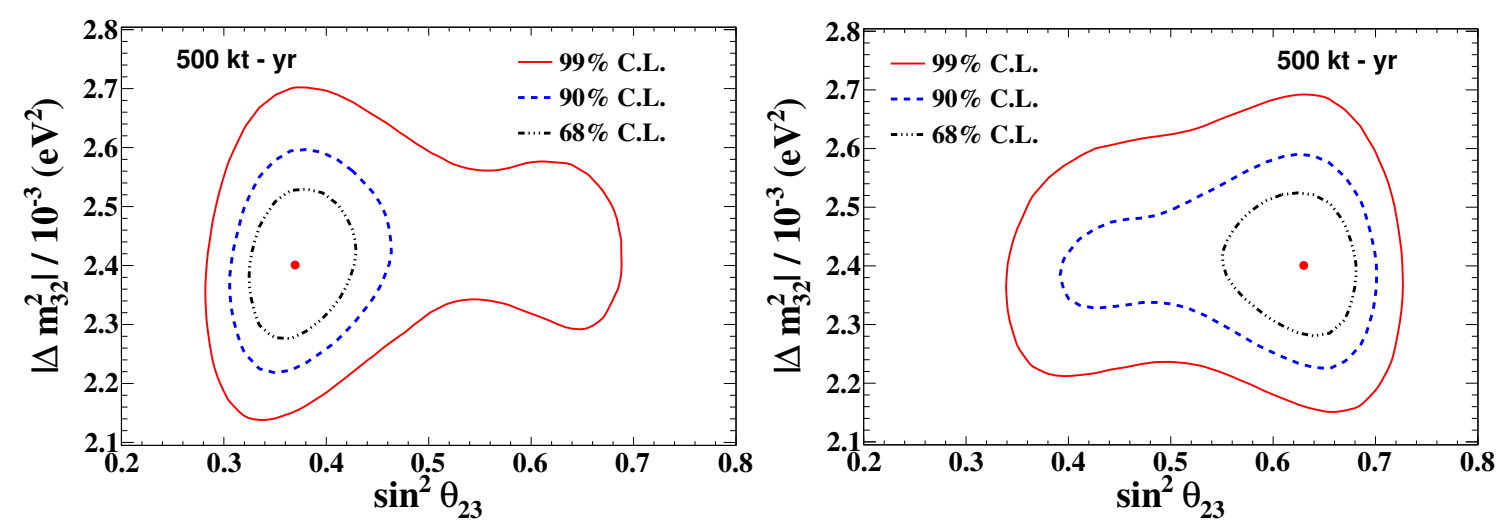

Figure 12. $\Delta \chi_{\text {ICAL-PM }}^{2}$ contours at $68 \%, 90 \%$, and $99 \%$ confidence levels (2 dof) in $\sin ^{2} \theta_{23}-\left|\Delta m_{32}^{2}\right|$ plane, for $\sin ^{2} \theta_{23}$ (true) $=0.37$ (left panel) and $\sin ^{2} \theta_{23}$ (true) $=0.63$ (right panel) after including the hadron energy information. We assume NH as the true hierarchy. The true choices of the parameters have been marked with a dot.
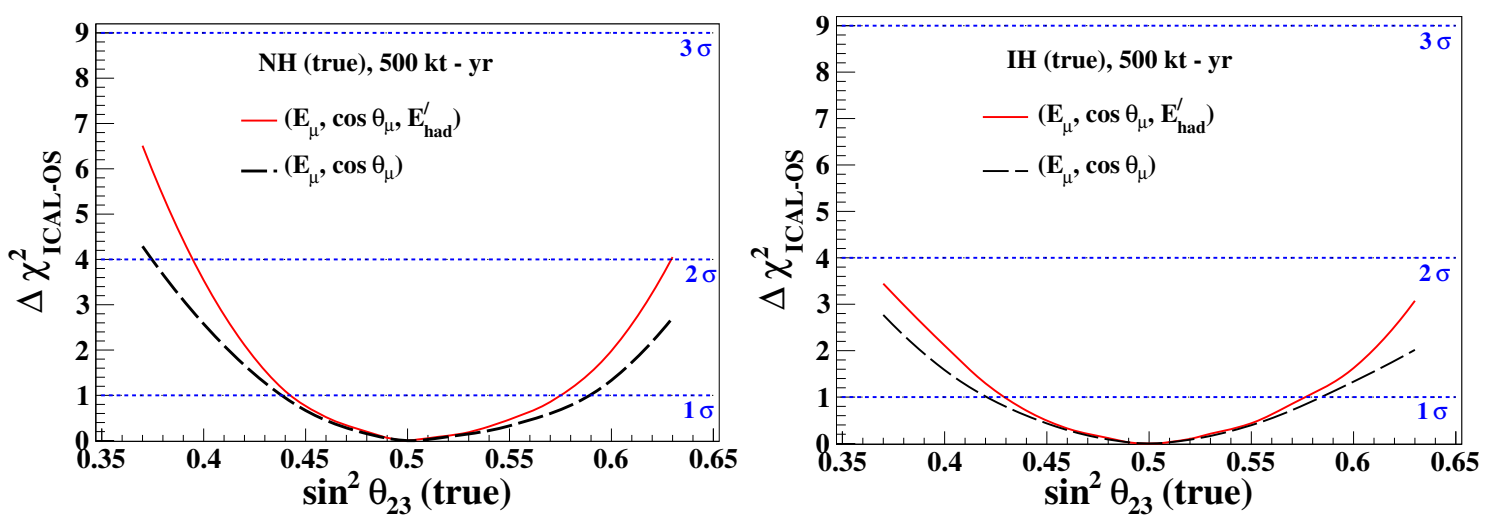

Figure 13. $\Delta \chi_{\text {ICAL-OS }}^{2}$ for octant discovery potential as a function of true $\sin ^{2} \theta_{23}$. The left panel (right panel) assumes $\mathrm{NH}(\mathrm{IH})$ as true hierarchy. The line labelled $\left(E_{\mu}, \cos \theta_{\mu}\right)$ denotes results without including hadron information, while the line labelled $\left(E_{\mu}, \cos \theta_{\mu}, E_{\text {had }}^{\prime}\right)$ denotes improved results after including hadron energy information. ICAL exposure of $500 \mathrm{kt}-\mathrm{yr}$ is considered.

Here $\chi_{\text {ICAL }}^{2}\left(\right.$ true octant) and $\chi_{\text {ICAL }}^{2}$ (false octant) are obtained by performing a fit to the "observed" data assuming the true octant and wrong octant, respectively. Here with the statistical fluctuations suppressed, $\chi_{\mathrm{ICAL}}^{2}($ true octant $) \approx 0$. For each given value of $\theta_{23}$ (true), we marginalize over all the allowed values of $\theta_{23}$ in the opposite octant, including the maximal mixing value. We also marginalize $\Delta \chi_{\text {ICAL-OS }}^{2}$ over the true choices of mass hierarchy in addition to the oscillation parameters mentioned in 2.3. The statistical significance for ruling out the wrong octant is represented in terms of $n \sigma$, where $n \equiv \sqrt{\Delta \chi_{\mathrm{ICAL}-\mathrm{OS}}^{2}}$.

Figure 13 shows the sensitivity of ICAL to the identification of the $\theta_{23}$ octant, with and without including the hadron energy information. It may be observed that a $2 \sigma$ identification of the octant is possible with the $500 \mathrm{kt}$-yr INO data alone only when the true hierarchy is $\mathrm{NH}$ and the true octant is LO. In this case, without using the hadron energy information one can get a $2 \sigma$ identification only when $\sin ^{2} \theta_{23}($ true $)<0.375$, which 
is almost close to the present $3 \sigma$ bound. With the addition of hadron energy information, this task is possible as long as $\sin ^{2} \theta_{23}$ (true) $<0.395$. If the true octant is $\mathrm{HO}$ or the true mass hierarchy is inverted, then the discrimination of $\theta_{23}$ octant with the ICAL data alone becomes rather difficult. In case of $\mathrm{NH}(\mathrm{IH})$, neutrino (antineutrino) events are mostly affected by the Earth's matter effect and give vital clues towards the octant of $\theta_{23}$. Since the statistical strength of atmospheric neutrino events is higher compared to antineutrino events, the octant sensitivity is better for $\mathrm{NH}$ compared to $\mathrm{IH}$. We have checked that these observations are not much sensitive to the true value of $\theta_{13}$. A variation of $\sin ^{2} 2 \theta_{13}$ (true) in the range 0.09 to 0.11 changes the values of $\Delta \chi_{\text {ICAL-OS }}^{2}$ only marginally. Clearly, the octant discrimination becomes more and more difficult as the true value of $\sin ^{2} \theta_{23}$ moves close to the maximal mixing. A combination of atmospheric as well as long-baseline experiments is needed to make this measurement [97-100].

\section{Summary and concluding remarks}

The main aim of the upcoming ICAL experiment at INO is to determine the mass hierarchy of neutrinos by observing the atmospheric neutrinos and exploiting the Earth matter effects on their oscillations. The major advantage of the ICAL detector is that it is well-tuned to detecting muons in the $\mathrm{GeV}$ range with a high efficiency, and reconstructing their energy and direction with a high precision. It can also identify the charge of the muons, which allows it to distinguish between an incoming $\nu_{\mu}$ and $\bar{\nu}_{\mu}$, a capability that is beyond the reach of other large atmospheric neutrino experiments. Because of these features the focus of the analyses for determining the physics reach of ICAL has so far been on exploiting the high-precision information on muon momenta.

However, a large detector like ICAL with its calorimetric properties is also capable of measuring the hadron energy, which may be parameterized in terms of the observable $E_{\text {had }}^{\prime} \equiv E_{\nu}-E_{\mu}$ through a hadron hit calibration procedure. In this paper, we present the enhancement in the physics reach of this experiment brought in by taking into account the combined information in muon momentum and hadron energy in each event. We focus on the identification of mass hierarchy and the precision measurements of atmospheric neutrino mixing parameters.

The additional information we seek is contained not only in the hadron energy distribution of events, but also in the correlations between the hadron energy and muon momentum in each event. For example, by using both $E_{\text {had }}^{\prime}$ and $E_{\mu}$ as observables, we indirectly probe the incoming neutrino energy. However a naive addition of these two to reconstruct the neutrino energy would lose the advantage of precise muon energy determination, and hence our analysis goes beyond that, by treating both these observables separately for each event. Indeed, when the muon and hadron information is combined on an event-by-event basis, one also gets access to what fraction of energy of the incoming neutrino is carried by the muon. This correlated muon and hadron information is what we try to extract in this analysis.

We adopt a binning scheme in the observables $\left(E_{\mu}, \cos \theta_{\mu}, E_{\text {had }}^{\prime}\right)$, where we divide the events in $10 E_{\mu}$ bins, $21 \cos \theta_{\mu}$ bins, and $4 E_{\text {had }}^{\prime}$ bins. We have used a relatively coarse 
binning scheme since we would like to have sufficient number of events in all bins. Since the hadron energy resolution at ICAL is not as precise as that for the muon, the number of $E_{\text {had }}^{\prime}$ bins has been chosen to be small. The non-uniform bins are such that the features relevant to the oscillations and matter effects are retained. We demonstrate that such an analysis yields marked improvements over the analyses that use muon information alone.

Adding the hadron energy information to the muon information, we find a significant enhancement in the capability of ICAL to determine the neutrino mass hierarchy. For the benchmark values of oscillation parameters, which are close to the current best-fit values, ICAL can determine the neutrino mass hierarchy with a significance of $\Delta \chi_{\text {ICAL-MH }}^{2} \approx 9$ with $500 \mathrm{kt}-y \mathrm{r}$ exposure. This is an improvement of more than $40 \%$ over the analysis that uses only muon information. This also implies that the same value of $\Delta \chi_{\text {ICAL-MH }}^{2}$ can be achieved with $40 \%$ less exposure when the correlated hadron information is added. We have also checked that the results with hadron energy are superior by about $35 \%$ even when a finer binning scheme $-20 E_{\nu}$ bins and $80 \cos \theta_{\mu}$ bins - is adopted for the analysis that uses only muon information. Depending on the true values of the oscillation parameters, the $\Delta \chi_{\mathrm{ICAL}-\mathrm{MH}}^{2}$ value varies between 7 and 12 , for an exposure of $500 \mathrm{kt}-\mathrm{yr}$. This is a crucial improvement, given that the main aim of the ICAL experiment is the identification of mass hierarchy.

We also demonstrate that the atmospheric neutrino mixing parameters can be measured more precisely by the inclusion of hadron energy information. Addition of hadron energy information improves the $\sin ^{2} \theta_{23}$ precision marginally from $14 \%$ to $12 \%$. However the precision on $\left|\Delta m_{32}^{2}\right|$ improves remarkably, from $4.2 \%$ to $2.9 \%$, even when the former has been obtained with the finer binning mentioned above. This may be attributed to a better determination of the original neutrino energy, and hence a better determination of the leading term in the muon survival probability that oscillates as $\sin ^{2}\left(\Delta m_{32}^{2} L / E_{\nu}\right)$. With the inclusion of hadron energy information, the expected precision on $\left|\Delta m_{32}^{2}\right|$ from ICAL after $500 \mathrm{kt}$-yr exposure is much better than the current reach of Super-Kamiokande; it is comparable to that obtained from MINOS, or with the current T2K data.

As far as the discrimination of $\theta_{23}$ octant is concerned, the hadron information increases the range of true $\theta_{23}$ values for which, say, a $2 \sigma$ discrimination is possible. However, such a discrimination would be possible with $500 \mathrm{kt}$-yr exposure only with true $\mathrm{NH}$ and and $\sin ^{2} \theta_{23}$ (true) $<0.395$. For higher values of $\theta_{23}$, the reach of ICAL alone is still limited.

It is clear from the above results that the inclusion of correlated hadron energy information improves oscillation physics sensitivities in almost all areas. However a few caveats are in order while interpreting the final numbers. We use the ICAL detector response to the muons and hadrons, as obtained by the INO collaboration. Given the current status of the understanding of the ICAL detector response obtained from simulations, we have had to make certain assumptions. For example, we assume that the muon track and the hadron shower can be separated completely in all events. We also neglect the background hits, noise, multiple hits, and assume that they do not affect the hadron response of the detector. As the understanding of the detector improves, including the reconstruction for muons and hadrons and the separation of hits due to them, the physics reach could be affected. However, this paper demonstrates quantitatively that, with the same conditions 
and assumptions, the inclusion of event-by-event hadron energy information in the analysis increases the reach for mass hierarchy identification and $\left|\Delta m_{32}^{2}\right|$ precision by a significant amount.

We expect this analysis procedure to become the preferred one for future analyses of ICAL physics reach. However it still does not extract all possible information contained in the events, for example the information in the hit pattern of hadron shower remains unexploited. A better understanding of the hadron response of the detector, and development of algorithms to use the hadron data efficiently would be crucial in making the most of the data that would be available from ICAL.

\section{Acknowledgments}

This work is a part of the ongoing effort of INO-ICAL collaboration to study various physics potentials of the proposed ICAL detector. Many members of the collaboration have contributed for the completion of this work. We are very grateful to K. Bhattacharya, G. Majumder and A. Redij for their developmental work on the ICAL detector simulation package. In addition, we thank S. Choubey, A. Ghosh, N. Mondal, D. Indumathi, S. Uma Sankar, S. Goswami, N. Sinha, P. Ghoshal, and M. Naimuddin for intensive discussions on the oscillation analysis in regular meetings. We would also like to thank V.M. Datar, R. Gandhi, and A. Raychaudhuri for their useful comments on the paper. S.K.A. would like to acknowledge M. Honda for useful communications. M.M.D. acknowledges the support from the Department of Atomic Energy (DAE) and the Department of Science and Technology (DST), Government of India. T.T. thanks Costas Andreopoulos for discussions on the neutrino event generators, and Tata Institute of Fundamental Research for their extended support. S.K.A. acknowledges the support from DST/INSPIRE Research Grant [IFA-PH12], Department of Science and Technology, India. A.D. acknowledges partial support from the European Union FP7 ITN INVISIBLES (Marie Curie Actions, PITN-GA-2011-289442).

Open Access. This article is distributed under the terms of the Creative Commons Attribution License (CC-BY 4.0), which permits any use, distribution and reproduction in any medium, provided the original author(s) and source are credited.

\section{References}

[1] DAYA BAy collaboration, F.P. An et al., Spectral measurement of electron antineutrino oscillation amplitude and frequency at Daya Bay, Phys. Rev. Lett. 112 (2014) 061801 [arXiv: 1310.6732] [INSPIRE].

[2] DAYA BAy collaboration, F.P. An et al., Improved Measurement of Electron Antineutrino Disappearance at Daya Bay, Chin. Phys. C 37 (2013) 011001 [arXiv:1210.6327] [INSPIRE].

[3] RENO collaboration, J.K. Ahn et al., Observation of Reactor Electron Antineutrino Disappearance in the RENO Experiment, Phys. Rev. Lett. 108 (2012) 191802 [arXiv: 1204.0626] [INSPIRE]. 
[4] DOUBLE-CHOOZ collaboration, Y. Abe et al., Indication for the disappearance of reactor electron antineutrinos in the Double CHOOZ experiment, Phys. Rev. Lett. 108 (2012) 131801 [arXiv:1112.6353] [INSPIRE].

[5] Double CHOOZ collaboration, Y. Abe et al., Reactor electron antineutrino disappearance in the Double CHOOZ experiment, Phys. Rev. D 86 (2012) 052008 [arXiv:1207.6632] [INSPIRE].

[6] MINOS collaboration, P. Adamson et al., Electron neutrino and antineutrino appearance in the full MINOS data sample, Phys. Rev. Lett. 110 (2013) 171801 [arXiv:1301.4581] [INSPIRE].

[7] T2K collaboration, K. Abe et al., Observation of Electron Neutrino Appearance in a Muon Neutrino Beam, Phys. Rev. Lett. 112 (2014) 061802 [arXiv:1311.4750] [InSPIRE].

[8] T2K collaboration, K. Abe et al., Evidence of Electron Neutrino Appearance in a Muon Neutrino Beam, Phys. Rev. D 88 (2013) 032002 [arXiv: 1304.0841] [INSPIRE].

[9] S. Pascoli and T. Schwetz, Prospects for neutrino oscillation physics, Adv. High Energy Phys. 2013 (2013) 503401.

[10] S.K. Agarwalla, S. Prakash and S. Uma Sankar, Exploring the three flavor effects with future superbeams using liquid argon detectors, JHEP 03 (2014) 087 [arXiv: 1304.3251] [INSPIRE].

[11] S.K. Agarwalla, Physics Potential of Long-Baseline Experiments, Adv. High Energy Phys. 2014 (2014) 457803 [arXiv: 1401.4705] [INSPIRE].

[12] H. Minakata, Neutrino Physics Now and in the Near Future, arXiv:1403.3276 [INSPIRE].

[13] C.H. Albright and M.-C. Chen, Model Predictions for Neutrino Oscillation Parameters, Phys. Rev. D 74 (2006) 113006 [hep-ph/0608137] [INSPIRE].

[14] L. Wolfenstein, Neutrino Oscillations in Matter, Phys. Rev. D 17 (1978) 2369 [InSPIRE].

[15] L. Wolfenstein, Neutrino Oscillations and Stellar Collapse, Phys. Rev. D 20 (1979) 2634 [INSPIRE].

[16] V.D. Barger, K. Whisnant, S. Pakvasa and R.J.N. Phillips, Matter Effects on Three-Neutrino Oscillations, Phys. Rev. D 22 (1980) 2718 [InSPIRE].

[17] S.P. Mikheev and A.Y. Smirnov, Resonance Amplification of Oscillations in Matter and Spectroscopy of Solar Neutrinos, Sov. J. Nucl. Phys. 42 (1985) 913 [Yad. Fiz. 42 (1985) 1441] [INSPIRE].

[18] S.P. Mikheev and A.Y. Smirnov, Resonant amplification of neutrino oscillations in matter and solar neutrino spectroscopy, Nuovo Cim. C 9 (1986) 17 [INSPIRE].

[19] M. Blennow and A.Y. Smirnov, Neutrino propagation in matter, Adv. High Energy Phys. 2013 (2013) 972485 [arXiv: 1306.2903] [INSPIRE].

[20] A.Y. Smirnov, Neutrino mass hierarchy and matter effects, arXiv:1312.7309 [INSPIRE].

[21] M. Blennow, P. Coloma, P. Huber and T. Schwetz, Quantifying the sensitivity of oscillation experiments to the neutrino mass ordering, JHEP 03 (2014) 028 [arXiv:1311.1822] [INSPIRE].

[22] T2K collaboration, Y. Itow et al., The JHF-Kamioka neutrino project, hep-ex/0106019 [INSPIRE]. 
[23] T2K collaboration, K. Abe et al., The T2K Experiment, Nucl. Instrum. Meth. A 659 (2011) 106 [arXiv: 1106.1238] [INSPIRE].

[24] D.S. Ayres et al., Letter of Intent to build an Off-axis Detector to study $\nu_{\mu} \rightarrow \nu_{e}$ oscillations with the NuMI Neutrino Beam, hep-ex/0210005 [INSPIRE].

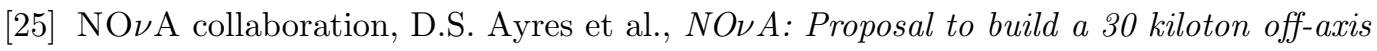
detector to study $\nu_{\mu} \rightarrow \nu_{e}$ oscillations in the NuMI beamline, hep-ex/0503053 [INSPIRE].

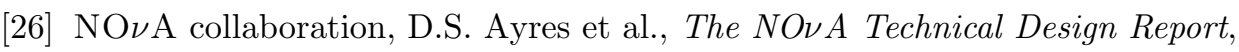
FERMILAB-DESIGN-2007-01 [INSPIRE].

[27] P. Huber, M. Lindner, T. Schwetz and W. Winter, First hint for CP-violation in neutrino oscillations from upcoming superbeam and reactor experiments, JHEP 11 (2009) 044 [arXiv: 0907.1896] [INSPIRE].

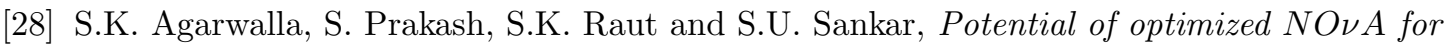
large $\theta_{13} \& 3$ combined performance with a LArTPC \& T2K, JHEP 12 (2012) 075 [arXiv: 1208.3644] [INSPIRE].

[29] M.V. Diwan et al., Very long baseline neutrino oscillation experiments for precise measurements of mixing parameters and CP-violating effects, Phys. Rev. D 68 (2003) 012002 [hep-ph/0303081] [INSPIRE].

[30] V. Barger et al., Report of the US long baseline neutrino experiment study, arXiv:0705.4396 [INSPIRE].

[31] P. Huber and J. Kopp, Two experiments for the price of one? The role of the second oscillation maximum in long baseline neutrino experiments, JHEP 03 (2011) 013 [Erratum ibid. 1105 (2011) 024] [arXiv: 1010.3706] [INSPIRE].

[32] LBNE collaboration, T. Akiri et al., The 2010 Interim Report of the Long-Baseline Neutrino Experiment Collaboration Physics Working Groups, arXiv:1110.6249 [INSPIRE].

[33] LBNE collaboration, C. Adams et al., The Long-Baseline Neutrino Experiment: Exploring Fundamental Symmetries of the Universe, arXiv:1307.7335 [INSPIRE].

[34] D. Autiero et al., Large underground, liquid based detectors for astro-particle physics in Europe: Scientific case and prospects, JCAP 11 (2007) 011 [arXiv:0705.0116] [INSPIRE].

[35] A. Rubbia, A CERN-Based High-Intensity High-Energy Proton Source for Long baseline Neutrino Oscillation Experiments with Next-Generation Large Underground detectors for Proton Decay Searches and Neutrino Physics and Astrophysics, arXiv:1003.1921 [INSPIRE].

[36] LAGUNA collaboration, D. Angus et al., The LAGUNA design study: Towards giant liquid based underground detectors for neutrino physics and astrophysics and proton decay searches, arXiv: 1001.0077 [INSPIRE].

[37] LAGUNA collaboration, A. Rubbia, The LAGUNA design study: Towards giant liquid based underground detectors for neutrino physics and astrophysics and proton decay searches, Acta Phys. Polon. B 41 (2010) 1727 [InSPIRE].

[38] S.K. Agarwalla, T. Li and A. Rubbia, An Incremental approach to unravel the neutrino mass hierarchy and CP-violation with a long-baseline Superbeam for large $\theta_{13}, J H E P 05$ (2012) 154 [arXiv:1109.6526] [INSPIRE].

[39] A. Stahl et al., Expression of Interest for a very long baseline neutrino oscillation experiment (LBNO), CERN-SPSC-2012-021 [INSPIRE]. 
[40] LAGUNA-LBNO collaboration, S.K. Agarwalla et al., The mass-hierarchy and $C P$-violation discovery reach of the LBNO long-baseline neutrino experiment, JHEP 05 (2014) 094 [arXiv: 1312.6520] [INSPIRE].

[41] Y.-F. Li, Overview of the Jiangmen Underground Neutrino Observatory (JUNO), Int. J. Mod. Phys. Conf. Ser. 31 (2014) 1460300 [arXiv:1402.6143] [InSPIRE].

[42] RENO-50 collaboration, in International Workshop on RENO-50 toward Neutrino Mass Hierarchy, Seoul National University, Korea, 13-14 June 2013 and online at http://home.kias.re.kr/MKG/h/reno50/.

[43] E.K. Akhmedov, Parametric resonance of neutrino oscillations and passage of solar and atmospheric neutrinos through the earth, Nucl. Phys. B 538 (1999) 25 [hep-ph/9805272] [INSPIRE].

[44] E.K. Akhmedov, A. Dighe, P. Lipari and A.Y. Smirnov, Atmospheric neutrinos at Super-Kamiokande and parametric resonance in neutrino oscillations, Nucl. Phys. B 542 (1999) 3 [hep-ph/9808270] [INSPIRE].

[45] M.V. Chizhov and S.T. Petcov, New conditions for a total neutrino conversion in a medium, Phys. Rev. Lett. 83 (1999) 1096 [hep-ph/9903399] [INSPIRE].

[46] M.C. Banuls, G. Barenboim and J. Bernabeu, Medium effects for terrestrial and atmospheric neutrino oscillations, Phys. Lett. B 513 (2001) 391 [hep-ph/0102184] [INSPIRE].

[47] R. Gandhi, P. Ghoshal, S. Goswami, P. Mehta and S.U. Sankar, Large matter effects in $\nu_{\mu} \rightarrow \nu_{\tau}$ oscillations, Phys. Rev. Lett. 94 (2005) 051801 [hep-ph/0408361] [INSPIRE].

[48] V. Barger et al., Neutrino mass hierarchy and octant determination with atmospheric neutrinos, Phys. Rev. Lett. 109 (2012) 091801 [arXiv:1203.6012] [INSPIRE].

[49] IceCube-PINGU collaboration, M.G. Aartsen et al., Letter of Intent: The Precision IceCube Next Generation Upgrade (PINGU), arXiv:1401.2046 [INSPIRE].

[50] KM3NeT collaboration, U.F. Katz, The ORCA Option for KM3NeT, PoS (NEUTEL 2013) 057 [arXiv: 1402.1022] [INSPIRE].

[51] E.K. Akhmedov, S. Razzaque and A.Y. Smirnov, Mass hierarchy, 2-3 mixing and CP-phase with Huge Atmospheric Neutrino Detectors, JHEP 02 (2013) 082 [Erratum ibid. 1307 (2013) 026] [arXiv: 1205.7071] [INSPIRE].

[52] S.K. Agarwalla, T. Li, O. Mena and S. Palomares-Ruiz, Exploring the Earth matter effect with atmospheric neutrinos in ice, arXiv:1212.2238 [INSPIRE].

[53] D. Franco et al., Mass hierarchy discrimination with atmospheric neutrinos in large volume ice/water Cherenkov detectors, JHEP 04 (2013) 008 [arXiv: 1301.4332] [INSPIRE].

[54] M. Ribordy and A.Y. Smirnov, Improving the neutrino mass hierarchy identification with inelasticity measurement in PINGU and ORCA, Phys. Rev. D 87 (2013) 113007 [arXiv: 1303.0758] [INSPIRE].

[55] W. Winter, Neutrino mass hierarchy determination with IceCube-PINGU, Phys. Rev. D 88 (2013) 013013 [arXiv:1305.5539] [INSPIRE].

[56] M. Blennow and T. Schwetz, Determination of the neutrino mass ordering by combining PINGU and Daya Bay II, JHEP 09 (2013) 089 [arXiv:1306.3988] [INSPIRE].

[57] S.-F. Ge, K. Hagiwara and C. Rott, A Novel Approach to Study Atmospheric Neutrino Oscillation, JHEP 06 (2014) 150 [arXiv:1309.3176] [INSPIRE]. 
[58] S.-F. Ge and K. Hagiwara, Physics Reach of Atmospheric Neutrino Measurements at PINGU, JHEP 09 (2014) 024 [arXiv:1312.0457] [INSPIRE].

[59] India-based Neutrino Observatory (INO), http://www.ino.tifr.res.in/ino/.

[60] INO collaboration, M.S. Athar et al., India-based Neutrino Observatory: Project Report. Volume I, INO-2006-01 [INSPIRE].

[61] A. Chatterjee et al., A Simulations Study of the Muon Response of the Iron Calorimeter Detector at the India-based Neutrino Observatory, 2014 JINST 9 P07001 [arXiv:1405.7243] [INSPIRE].

[62] S.K. Agarwalla, Status and prospects of ino, talk given at the NNN 2013 Workshop, Kavli IPMU, Japan, 11-13 November 2013 and online at http://indico.ipmu.jp/indico/conferenceDisplay.py?confId $=17$.

[63] S. Palomares-Ruiz and S.T. Petcov, Three-neutrino oscillations of atmospheric neutrinos, $\theta_{13}$, neutrino mass hierarchy and iron magnetized detectors, Nucl. Phys. B 712 (2005) 392 [hep-ph/0406096] [INSPIRE].

[64] D. Indumathi and M.V.N. Murthy, A Question of hierarchy: Matter effects with atmospheric neutrinos and anti-neutrinos, Phys. Rev. D 71 (2005) 013001 [hep-ph/0407336] [INSPIRE].

[65] S.T. Petcov and T. Schwetz, Determining the neutrino mass hierarchy with atmospheric neutrinos, Nucl. Phys. B 740 (2006) 1 [hep-ph/0511277] [INSPIRE].

[66] A. Samanta, The Mass hierarchy with atmospheric neutrinos at INO, Phys. Lett. B 673 (2009) 37 [hep-ph/0610196] [INSPIRE].

[67] R. Gandhi, P. Ghoshal, S. Goswami, P. Mehta, S.U. Sankar and S. Shalgar, Mass Hierarchy Determination via future Atmospheric Neutrino Detectors, Phys. Rev. D 76 (2007) 073012 [arXiv: 0707.1723] [INSPIRE].

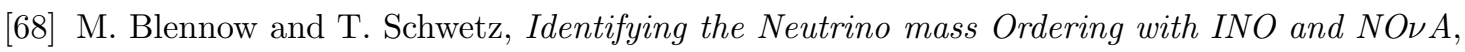
JHEP 08 (2012) 058 [Erratum ibid. 1211 (2012) 098] [arXiv:1203.3388] [INSPIRE].

[69] A. Ghosh, T. Thakore and S. Choubey, Determining the Neutrino Mass Hierarchy with INO,

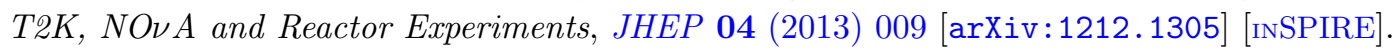

[70] T. Thakore, A. Ghosh, S. Choubey and A. Dighe, The Reach of INO for Atmospheric Neutrino Oscillation Parameters, JHEP 05 (2013) 058 [arXiv:1303.2534] [INSPIRE].

[71] R.N. Mohapatra and A.Y. Smirnov, Neutrino Mass and New Physics, Ann. Rev. Nucl. Part. Sci. 56 (2006) 569 [hep-ph/0603118] [INSPIRE].

[72] C.H. Albright, A. Dueck and W. Rodejohann, Possible Alternatives to Tri-bimaximal Mixing, Eur. Phys. J. C 70 (2010) 1099 [arXiv: 1004.2798] [InSPIRE].

[73] S.F. King and C. Luhn, Neutrino Mass and Mixing with Discrete Symmetry, Rept. Prog. Phys. 76 (2013) 056201 [arXiv: 1301.1340] [INSPIRE].

[74] G.L. Fogli and E. Lisi, Tests of three flavor mixing in long baseline neutrino oscillation experiments, Phys. Rev. D 54 (1996) 3667 [hep-ph/9604415] [INSPIRE].

[75] A. Chatterjee, R. Gandhi and J. Singh, Probing Lorentz and CPT Violation in a Magnetized Iron Detector using Atmospheric Neutrinos, JHEP 06 (2014) 045 [arXiv:1402.6265] [INSPIRE]. 
[76] M. Ghosh, P. Ghoshal, S. Goswami and S.K. Raut, Can atmospheric neutrino experiments provide the first hint of leptonic CP-violation?, Phys. Rev. D 89 (2014) 011301 [arXiv: 1306.2500] [INSPIRE].

[77] M.M. Devi et al., Hadron energy response of the Iron Calorimeter detector at the India-based Neutrino Observatory, 2013 JINST 8 P11003 [arXiv:1304.5115] [INSPIRE].

[78] A. Samanta and A.Y. Smirnov, The 2-3 mixing and mass split: atmospheric neutrinos and magnetized spectrometers, JHEP 07 (2011) 048 [arXiv: 1012.0360] [INSPIRE].

[79] D. Kaur, M. Naimuddin and S. Kumar, Neutrino oscillation analysis for ino-ical detector, in preparation, (2014).

[80] A. Ghosh and S. Choubey, Measuring the Mass Hierarchy with Muon and Hadron Events in Atmospheric Neutrino Experiments, JHEP 10 (2013) 174 [arXiv:1306.1423] [INSPIRE].

[81] D. Casper, The Nuance neutrino physics simulation and the future, Nucl. Phys. Proc. Suppl. 112 (2002) 161 [hep-ph/0208030] [INSPIRE].

[82] M. Honda, T. Kajita, K. Kasahara and S. Midorikawa, Improvement of low energy atmospheric neutrino flux calculation using the JAM nuclear interaction model, Phys. Rev. D 83 (2011) 123001 [arXiv: 1102.2688] [INSPIRE].

[83] R. Bayes et al., The Golden Channel at a Neutrino Factory revisited: improved sensitivities from a Magnetised Iron Neutrino Detector, Phys. Rev. D 86 (2012) 093015 [arXiv: 1208.2735] [INSPIRE].

[84] M. Sajjad Athar, M. Honda, T. Kajita, K. Kasahara and S. Midorikawa, Atmospheric neutrino flux at INO, South Pole and Pyhasalmi, Phys. Lett. B 718 (2013) 1375 [arXiv: 1210.5154] [INSPIRE].

[85] D.V. Forero, M. Tortola and J.W.F. Valle, Neutrino oscillations refitted, arXiv:1405.7540 [INSPIRE].

[86] NuFIT webpage, http://www.nu-fit.org/.

[87] M.C. Gonzalez-Garcia, M. Maltoni, J. Salvado and T. Schwetz, Global fit to three neutrino mixing: critical look at present precision, JHEP 12 (2012) 123 [arXiv:1209.3023] [INSPIRE].

[88] F. Capozzi, G.L. Fogli, E. Lisi, A. Marrone, D. Montanino and A. Palazzo, Status of three-neutrino oscillation parameters, circa 2013, Phys. Rev. D 89 (2014) 093018 [arXiv: 1312.2878] [INSPIRE].

[89] A. de Gouvêa, J. Jenkins and B. Kayser, Neutrino mass hierarchy, vacuum oscillations and vanishing $\left|U_{e 3}\right|$, Phys. Rev. D 71 (2005) 113009 [hep-ph/0503079] [INSPIRE].

[90] H. Nunokawa, S.J. Parke and R. Zukanovich Funchal, Another possible way to determine the neutrino mass hierarchy, Phys. Rev. D 72 (2005) 013009 [hep-ph/0503283] [INSPIRE].

[91] E.K. Akhmedov, R. Johansson, M. Lindner, T. Ohlsson and T. Schwetz, Series expansions for three flavor neutrino oscillation probabilities in matter, JHEP 04 (2004) 078 [hep-ph/0402175] [INSPIRE].

[92] G.L. Fogli, E. Lisi, A. Marrone, D. Montanino, A. Palazzo and A.M. Rotunno, Global analysis of neutrino masses, mixings and phases: entering the era of leptonic CP-violation searches, Phys. Rev. D 86 (2012) 013012 [arXiv:1205.5254] [INSPIRE].

[93] Super-Kamiokande collaboration, A. Himmel, Recent results from Super-Kamiokande, AIP Conf. Proc. 1604 (2014) 345 [arXiv:1310 .6677] [InSPIRE]. 
[94] MINOS collaboration, P. Adamson et al., Combined analysis of $\nu_{\mu}$ disappearance and $\nu_{\mu} \rightarrow \nu_{e}$ appearance in MINOS using accelerator and atmospheric neutrinos, Phys. Rev. Lett. 112 (2014) 191801 [arXiv:1403.0867] [INSPIRE].

[95] T2K collaboration, K. Abe et al., Precise Measurement of the Neutrino Mixing Parameter $\theta_{23}$ from Muon Neutrino Disappearance in an Off-axis Beam, Phys. Rev. Lett. 112 (2014) 181801 [arXiv: 1403.1532] [INSPIRE].

[96] S. Choubey and P. Roy, Probing the deviation from maximal mixing of atmospheric neutrinos, Phys. Rev. D 73 (2006) 013006 [hep-ph/0509197] [INSPIRE].

[97] A. Chatterjee, P. Ghoshal, S. Goswami and S.K. Raut, Octant sensitivity for large $\theta_{13}$ in atmospheric and long baseline neutrino experiments, JHEP 06 (2013) 010 [arXiv: 1302.1370] [INSPIRE].

[98] M. Ghosh, P. Ghoshal, S. Goswami and S.K. Raut, Synergies between neutrino oscillation experiments: an 'adequate' configuration for LBNO, JHEP 03 (2014) 094 [arXiv: 1308.5979] [INSPIRE].

[99] S. Choubey and A. Ghosh, Determining the Octant of $\theta_{23}$ with PINGU, T2K, NOLA and Reactor Data, JHEP 11 (2013) 166 [arXiv:1309.5760] [INSPIRE].

[100] S.K. Agarwalla, S. Choubey and A. Ghosh, Probing 2-3 neutrino mixing at ical-ino

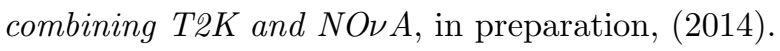

